\title{
Anabases
}

ANABASES Traditions et réceptions de l'Antiquité

$27 \mid 2018$

Varia

William H.F. ALTMAN

(éd.),

\section{Brill's Companion to the Reception of Cicero}

Amedeo Alessandro Raschieri

\section{(2) OpenEdition}

Edizione digitale

URL: http://journals.openedition.org/anabases/7180

DOI: 10.4000/anabases.7180

ISSN: 2256-9421

Editore

E.R.A.S.M.E.

\section{Edizione cartacea}

Data di pubblicazione: 1 avril 2018

Paginazione: 219-220

ISSN: 1774-4296

\section{Notizia bibliografica digitale}

Amedeo Alessandro Raschieri, «William H.F. altman(éd.),Brill's Companion to the Reception of Cicero», Anabases [Online], 27 | 2018, Messo online il 01 avril 2018, consultato il 20 janvier 2021. URL: http:// journals.openedition.org/anabases/7180 ; DOI: https://doi.org/10.4000/anabases.7180 


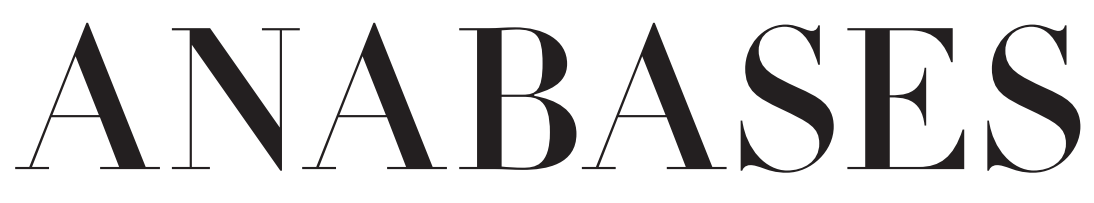

Traditions et Réceptions de l'Antiquité

$$
\begin{aligned}
& \text { No27 } \\
& 2018
\end{aligned}
$$

E.R.A.S.M.E.

Université Toulouse - Jean Jaurès 



\section{Sommaire}

$\mathrm{N}^{\circ} 27-2018$

\section{Historiographie et identités culturelles}

\section{Sébastien Cazalas}

“ et pour ce recite Valere... ». La place de l'Antiquité dans l'arsenal

intellectuel d'un grand prélat français du XVe siècle :

Jean Juvénal des Ursins (I388-I473) . . . . . . . . . . . . . . . . . . . II

Loic Marcou

La réception de l'Antiquité dans l'œuvre de la femme de lettres grecque

Rhéa Galanaki. . . . . . . . . . . . . . . . . . . . . .

\section{Traditions du patrimoine antique}

« Restituer Herculanum II. Des archives de fouilles aux restitutions 3D

Alexandra DARDENAY

Introduction : restituer l'espace domestique à Herculanum

grâce aux outils informatiques de reconstruction virtuelle ;

enjeux et problèmes. . . . . . . . . . . . . . . 4

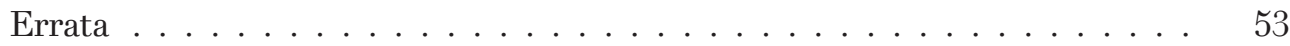

Carla Marotta

L’utilisation des données archivistiques dans l'enquête archéologique : nouvelles découvertes sur la maison d'Argos . . . . . . . . . . . . . . .

Emmanuelle Rosso

Restituer l'emplacement des statues dans les édifices d'Herculanum?

Problèmes de méthode . . . . . . . . . . . . . . . 7I 
Hélène Eristov, Marie-Laure Maraval

Restituer les décors perdus de la maison de Neptune et d'Amphitrite :

enquête, méthodes, résultats . . . . . . . . . . . . 9 9I

Maud Mulliez

Restauration numérique des peintures murales de la maison

de Neptune et Amphitrite à Herculanum : de l'expérimentation

matérielle à l'intégration des données dans un modèle 3D . . . . . . . Io3

\section{Archéologie des savoirs}

Nicolas SIRON

L'histoire de Philippidès d'Hérodote à Lucien.

Une incursion dans l'atelier du mythe . . . . . . . . . . . . . . . . Iog

Constantin Raios

Le coracin (xo@axîvos) du lac de Tibériade (F. Josèphe, B.J. III, 520) . . .

\section{Actualités et débats}

Claude Azıza

Antiquités parallèles (8). Le syndrome du sein droit . . . . . . . . . . . . I6I

Elisabeth DÉcultot

Winckelmann. Moderne Antike / Winckelmann. Modern Antiquity.

Exposition, Weimar, Neues Museum, 7 avril-2 juillet 20I7 . . . . . . . I67

\section{Lire, relire la bibliothèque des sciences de l'Antiquité}

Cyrielle LANDrea

Jérôme Carcopino et la mémoire perdue

de M. Valerius Messalla Corvinus (cos. 3г av. J.-C.) . . . . . . . . . . . . $\quad$ I77

Jérôme CARCopino

Notes biographiques sur M. Valerius Messala Corvinus . . . . . . . . . I I85

\section{L'atelier de l'histoire : chantiers historiographiques}

L'Atelier des doctorants (coordonné par Adeline Grand-Clément) (15)

Jonathan Pérez Mostazo

Cantabri aut vascones. La reception de la Antigüedad

en la cultura histórica vasca del siglo XIX . . . . . . . . . . . . . . . . . 209 


\section{Comptes rendus}

William H.F. Altman (éd.)

Brill's Companion to the Reception of Cicero (A. A. Raschieri). . . . . . . . 2 2I9

Anthony A. Barrett, Elaine Fantham, John C. Yardley

The Emperor Nero. A Guide to the Ancient Sources (É. Deniaux) . . . . . . 22I

Pascale Barthélémy et Violaine sébillotte cuchet (éd.)

Clio. Femmes, Genre, Histoire $\mathrm{n}^{\circ} 43$ : Citoyennetés (M. L. Napolitano) . . . 222

Frédéric Colin, Olivier Huck, Sylvie Vanseveren (éd.)

Interpretatio. Traduire l'altérité culturelle dans les civilisations

de l'Antiquité (Cl. Joncheray) . . . . . . . . . . . . . . . . . . . . 224

Angus Fletcher

Comic Democracies. From Ancient Athens

to the American Republic (A. de Crémoux). . . . . . . . . . . . . . . . . . 226

David Hernández de la Fuente

El despertar del alma. Dioniso y Ariadna: mito y misterio (A. Iriarte) . . . . 228

Ted Kaizer (éd.)

Religion, Society and Culture at Dura-Europos (P. G. Michelotto) . . . . . 229

Jacques Le Goff, Jean-Pierre Vernant

Dialogue sur l'histoire. Entretiens avec Emmanuel Laurentin (C. Lucci). . 23I

Justine Mac Connell, Edith Hall

Ancient Greek Myth in World Fiction since ig8g (A. de Crémoux) . . . . . . 233

Angelo Mazzocco, Marc Laureys (éd.)

ANew Sense of the Past. The Scholarship

of Biondo Flavio (I3g2-I463) (C. Lucci). . . . . . . . . . . . . . . . . . . . . . 234

Seth L. Schein

Homeric Epic and Its Reception. Interpretative Essays (S. Sistac) . . . . . . 236

Arbogast SснмiтT

Wie aufgeklärt ist die Vernunft der Aufklärung?

Eine Kritik aus aristotelischer Sicht (L. Calvié) . . . . . . . . . . . . . . . . 238

Richard Sorabu (éd.)

Aristotle Re-Interpreted. New Findings on Seven Hundred Years

of the Ancient Commentators (P. Butti de Lima) . . . . . . . . . . . . . . . 239

Laura SwiFT

Greek Tragedy. Themes and Contexts (G. Aujac) . . . . . . . . . . . . . . 24

Zara Martirosova TorLone

Vergil in Russia. National Identity and Classical Reception

(D. Millet-Gérard) . . . . . . . . . . . . . . . . . . 243 
Jürgen von UNGERN-STERNBERG

Les chers ennemis. Deutsche und französische

Altertumswissenschaftler in Rivalität und Zusammenarbeit (L. Calvié) . . 2444

Robert W. WALLACE

Reconstructing Damon: Music, Wisdom Teaching, and Politics

in Perikles'Athens (A. Brancacci) . . . . . . . . . . . . . . . . . . . . . 246

T. P. Wiseman

The Roman Audience : Classical Literature as Social History (C. Landrea) 249

Résumés . . . . . . . . . . . . . . . . . . . . . . . . . . 253

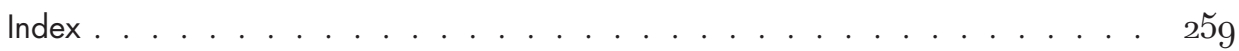




\section{Comptes rendus de lecture}



William H.F. Altman (éd.), Brill's Companion to the Reception of Cicero, Leiden - Boston, Brill, 2015, 402 p., I49 euros / ISBN 978-90-04-23526-7.

Chi cercherà una trattazione esaustiva sul problema della ricezione ciceroniana nel corso dei secoli, certo non sarà soddisfatto dal volume in esame. Chi invece vorrà trovare una guida per esplorare la questione, non potrà che considerare i saggi qui raccolti come un punto di riferimento fondamentale, soprattutto in vista di ulteriori approfondimenti. La regia di William H.F. Altman è riuscita a costruire un percorso che, nonostante l'assenza di un ordine cronologico, presenta in modo chiaro vari punti di vista inseriti in un orizzonte coerente.

In generale, si può osservare che il confronto con Cicerone ha trovato in ogni epoca le sue ragioni più profonde non tanto nell'interesse erudito ma soprattutto nell'urgenza della contemporaneità. Da ciò derivano le diverse prospettive, raggruppate in cinque sezioni, che strutturano il volume: il rapporto ambiguo tra imitazione e critica, lo sfondo politico delle riletture ciceroniane, gli sguardi focalizzati su Francia e Germania, le dinamiche bipartite di alcuni momenti della ricezione ciceroniana e, infine, una riflessione sui momenti in cui l'opera ciceroniana costituisce il fondamento della modernità filosofica e letteraria.

Dopo l'introduzione del curatore, il contributo di M. McLaughlin (Petrarch and Cicero. Adulation and Critical Distance) approfondisce la presenza ciceroniana nell'opera di Petrarca e conclude con la constatazione che l'umanista rappresenta il fondamento della fortuna di Cicerone nel mondo moderno. Il passo successivo porta a Montaigne: K. Eden (Cicero's Portion of Montaigne's Acclaim) riconosce l'influenza ciceroniana sull'autore francese non solo a livello filosofico ma anche nella costruzione retorica degli Essais. L'ultimo saggio della prima sezione riporta indietro nel tempo alla presenza di Cicerone nell'opera di Lattanzio (Lactantius as Christian Cicero, Cicero as Shadow-like Instructor): G. Kendeffy sottolinea che il pensatore cristiano considerava Cicerone come un terreno comune tra pagani e cristiani, funzionale alla dimostrazione che il paganesimo fosse una falsa versione del cristianesimo.

Si passa poi al ruolo di Cicerone nel Settecento inglese: R.G. Ingram (Conyers Middleton's Cicero. Enlightenment, Scholarship, and Polemic) mostra come in C. Middleton l'attenzione per la figura storica e il pensiero di Cicerone nascesse dalle aspre polemiche religiose del tempo. Con il contributo di C. J. Richard (Cicero and the American Founders) dalla religione si giunge alla politica e, in particolare, all'importanza di Cicerone nel sistema educativo americano settecentesco e nella fondazione del sistema costituzionale degli Stati Uniti d'America. Per chiudere la sezione, si torna ancora una volta all'antichità e alla ricostruzione di un ruolo politico per Cicerone che A. Dessler (Cicero's Quarrels. Reception and Modernity from Horace to Tacitus) riconosce nel Dialogus de oratoribus di Tacito e, in particolare, nella 
polemica tra antichi e moderni attraverso la tappa intermedia di Orazio.

Come esempi di ricezione ciceroniana in Francia sono scelti i casi di J. Derrida e J. Carcopino. P. A. Miller (Cicero Reads Derrida Reading Cicero. A Politics and a Friendship to Come) concentra la sua attenzione sul libro Politiques de l'amitié (1994) di Derrida, in cui il filosofo offre una lettura personale del De amicitia di Cicerone e mostra una profonda sintonia con il pensatore romano. Segue la riproposizione di un saggio di C. Lévy (Ancient Texts, Contemporary Stakes. J. Carcopino as Reader of Cicero's Letters), già pubblicato in francese nel 2006. Lo studioso, con grande passione politica e altrettanta sensibilità storica, mostra come il giudizio di J. Carcopino su Cicerone fosse influenzato dalle proprie vicende personali e dal suo ruolo pubblico durante il governo di Vichy.

Per quanto riguarda l'ambito tedesco con un'appendice nel mondo anglosassone, W.H.F.Altman (Cicero and the Fourth Triumvirate. Gruen, Syme, and Strasburger) individua una triade di studiosi E. S. Gruen, H. Strasburger, R. Syme che hanno negativamente condizionato l'interpretazione della figura storica di Cicerone nel Novecento attraverso la sua contrapposizione all'azione politica di Cesare. In seguito, E. Begemann (Damaged Go(o)ds. Cicero's Theological Triad in the Wake of German Historicism) evidenzia i limiti degli studi tedeschi sul pensiero teologico di Cicerone tra il tardo Ottocento e la prima metà del Novecento soprattutto in merito al giudizio sull'originalità del pensiero ciceroniano in confronto con le fonti greche.

Due sono i punti di vista sulle interpretazioni dicotomiche dell'opera ciceroniana. C. Bishop (Roman Plato or Roman Demosthenes? The Bifurcation of Cicero in Ancient Scholarship) analizza gli autori antichi che fornirono letture parziali di Cicerone come oratore (Quinto Asconio Pediano) o come filosofo (Macrobio).
J. O. Ward (What the Middle Ages Missed of Cicero, and Why), invece, si concentra sui lettori medioevali di Cicerone (in particolare Guglielmo di Malmesbury e Giovanni di Salisbury) e spiega perché, a fronte di una supervalutazione di opere retoriche come il De inventione o la Rhetorica ad Herennium, riconosciuta come autenticamente ciceroniana, non fosse attribuita altrettanta importanza alla produzione filosofica di Cicerone.

Il volume si chiude con due contributi che mettono in luce momenti in cui Cicerone diventa il modello per la riflessione filosofica e lo stile letterario. M. Sharpe (Cicero, Voltaire, and the Philosophes in the French Enlightenment), attraverso un'attenta lettura delle opere di Voltaire, mostra come Cicerone fosse un'autorità indiscussa anche per i pensatori dell'Illuminismo. Infine, J. DellaNeva (Following Their Own Genius. Debates on Ciceronianism in I6th-Century Italy) ripercorre la questione del ciceronianismo nell'umanesimo italiano attraverso il dibattito tra P. Bembo e G. Pico della Mirandola: quest'ultimo, sebbene prenda le distanze dagli imitatori pedissequi di Cicerone, risulta essere il più autenticamente ciceroniano.

Da questa rassegna, si conferma il giudizio iniziale di un volume ricco e articolato che accompagna il lettore attraverso i problemi fondamentali della ricezione ciceroniana tra antichità ed età contemporanea, non senza qualche incoerenza cronologica e forzatura contenutistica. Da ultimo, è opportuno sottolineare che, sebbene la bibliografia sia ampia e comprenda studi scritti nelle principali lingue europee, gli autori, tranne rare ma autorevoli eccezioni (E. Begemann, G. Kendeffy, C. Lévy), provengano per lo più dal mondo anglosassone e, in particolare, si senta la mancanza dei punti di vista spagnolo e italiano.

Amedeo Alessandro Raschieri Università degli Studi di Milano amedeo.raschieri@gmail.com 
Anthony A. Barrett, Elaine Fantham, John C. YARDLEY

The Emperor Nero. A Guide to the Ancient Sources, Princeton

Princeton University Press, 20I6, 3oo p., 35 dollars / IsBN 978069i5565ı4.

Le règne de Néron a toujours exercé une grande fascination. Les auteurs du livre ont voulu mettre à la disposition du public, et particulièrement des étudiants, un recueil de sources concernant le règne du dernier des Julio-Claudiens. Le guide des sources anciennes concernant le règne de Néron a été réalisé par Anthony A. Barrett, professeur de classics à l'université de Colombie britannique, Elaine Fantham, professeur de latin à l'université de Princeton, John C. Yardley, professeur de classics à l'université d'Ottawa. Leur entreprise est originale. Elle dépasse celle des habituels recueils de sources qui sont des morceaux choisis car le recensement des sources littéraires concernant ce règne vise à l'exhaustivité. Les textes latins et grecs ont été traduits par les trois auteurs qui ont ajouté des notes impressionnantes par leur qualité scientifique. Dix chapitres composent ce volume, précédés par une large introduction qui présente aussi les grands auteurs grecs et latins et une chronologie des événements principaux de la vie de Néron. Trois chapitres sont consacrés à l'empereur et à son arrivée au pouvoir, aux ennemis de l'intérieur ; ils sont suivis par deux chapitres traitant de la politique extérieure, en Parthie, en Bretagne et en Germanie; un chapitre est consacré ensuite au grand incendie de 64 et à ses conséquences en particulier sur la persécution des chrétiens, suivi par trois autres qui traitent des femmes de l'empereur Néron, des conspirations visant l'empereur, et de l'empereur Néron comme artiste et comme homme de scène (chapitre 9), suivi d'un dernier chapitre consacré à la mort de l'empereur.

Ces chapitres sont de véritables monographies ; par exemple le chapitre 4, intitulé
“Parthia », est une étude très complète sur les relations entreles Romains et les Parthes, revenant sur les périodes antérieures, examinant les événements année par année depuis l'année 54 jusqu'à l'année 63 (p. 77 à II7). Pour la Germanie, au chapitre 5 , la présentation du problème de la frontière se fonde surtout sur les textes des Annales de Tacite. Cependant une inscription (ILS 232) est citée à propos de Corbulon (p. II6); à la même page, une autre, très fragmentaire, est mentionnée curieusement avec comme référence CIL 6742 sans mention du tome du corpus, alors que le numéro du tome, le tome 3, aurait dû être ajouté puisque cette inscription a été trouvée en Arménie. Dans le même chapitre, le chapitre 5, sont recensées les sources littéraires concernant l'histoire de la Bretagne, avec des commentaires très abondants sur la révolte de Boudicca qui présentent même une recherche sur le nom de la reine Boudicca et des hypothèses sur sa tombe (p. 123). Nous pouvons noter aussi, entre autres, les qualités du chapitre 9, “l'empereur comme artiste et homme de scène ", écrit par Elaine Fantham, qui étudie les différents effets des performances de l'empereur « artiste » dans sa capitale à Rome, mais aussi à Naples et en Grèce. Il contient un riche développement (p. 25i et suivantes) sur l'admiration portée par les Grecs à Néron auxquels l'empereur proclame la liberté ainsi que la traduction d' une inscription gravée sur le mur de la chapelle Saint Georges à Acraephia (ILS 8794) avec trois documents distincts, l'édit de Néron rassemblant les Grecs à Corinthe, le discours prononcé par Néron à Corinthe pour proclamer la libération de la Grèce, le décret d'Épaminondas d'Acraephia, prêtre du culte impérial qui proposa de dédier un autel en l'honneur de Néron et de faire des offrandes en remerciement pour le geste exceptionnel de l'Empereur. À la page 253 est présenté un fac-similé du décret d'Épaminondas dont l'origine n'est pas mentionnée. Quelques documents épigraphiques sont ainsi apportés en com- 
plément du corpus littéraire avec aussi un papyrus, $P O x y$ VII, IO2I concernant l'arrivée au pouvoir de Néron, et une représentation figurée de Néron dans le Sebasteion d'Aphrodisias. Le dossier est complété par l'examen de neuf monnaies bien choisies et bien commentées, monnaies présentées avec les références au $R I C$ et provenant de la collection du site d'Andreas Pangerl: http://www.romancoins.info/. Il est possible de regretter l'absence d'un index des sources des documents littéraires et des autres documents non littéraires qui aurait pu constituer un complément très précieux à ce recueil et en rendre la consultation plus facile. L'exhaustivité de la documentation littéraire concernant le règne de Néron constitue la richesse de ce volume. La traduction en langue anglaise de celleci permet une approche plus aisée à ceux qui aujourd'hui maîtrisent mal les langues anciennes. La qualité des commentaires historiques, où prennent place des débats historiographiques récents, en font un ouvrage très utile.

\section{Élizabeth Deniaux \\ Université Paris-Nanterre eldeniaux@wanadoo.fr}

\author{
Pascale BarthéLÉmy et \\ Violaine SÉBILlotTE CuCHEt (éd.), \\ Clio. Femmes, Genre, Histoire n ${ }^{\circ} 43$ : \\ Citoyennetés, \\ Paris, Belin, 20I6, 332 p., \\ 26 euros / ISBN 978-2-70II-9852-I.
}

La rivista Clio intitola il numero $46 \mathrm{del}$ 2016 alle cittadinanze. L'indagine tematica è ripartita nelle sezioni Dossier, Regards complémentaires, Documents, Actualité de la recherche, Portrait, Varia. A partire dal mondo greco e romano, gli studi affrontano aspetti della questione 'cittadinanza' al femminile, declinati nella diversità delle prospettive e delle coordinate di tempo e spazio. Filo conduttore del volume, come nell'introduzione di Pascale Barthélémy e di Violaine Sebillotte Cuchet, è la riflessione sulla cittadinanza giuridica, nozione ripensata dagli studi di storia delle donne e di genere fino alla definizione di "cittadinanze" plurime, connesse alla condivisione pubblica di aspetti della vita della comunità: lingua, pratiche sociali, riti e incarichi religiosi. Nell'indagare il legame tra cittadinanza e individuo, tra cittadinanza e nazionalità, ci si interroga inoltre sulla combinazione di più appartenenze, che interessano aspetti sociali, politici, economici, culturali, religiosi.

Introduce la sezione Dossier il saggio di Aude Chatelard su esclusione giuridica e cittadinanza delle donne romane di età repubblicana: le 'cittadinanze' femminili si aprono alla dimensione sociale e del vissuto soggettivo nel mito, negli incarichi e nei riti della religione statale, nonostante le donne non condividano la cittadinanza giuridica per infirmitas sexus. Si rintracciano forme di partecipazione pubblica e di appartenenza politica in categorie di vedove, nei sacerdozi femminili, negli interventi diretti e incisivi delle donne nelle contiones maschili. Per l'Italia medievale e moderna, Simona Feci esplora le prospettive della cittadinanza e della mobilità femminili. Le donne declinano le loro 'cittadinanze' mediante forme di appartenenza peculiari: sostegno a confraternite femminili per conseguire tutele, concessioni, forme di integrazione proprie della cittadinanza giuridica; attività lavorativa e carico fiscale pro coniuge delle vedove; matrimoni e mantenimento, se straniere, di legami con la comunità di origine. In una diversa prospettiva, Linda Guerry studia la cittadinanza statale-nazionale delle donne sposate che ottengono la doppia nazionalità grazie alla convenzione dell'assemblea generale dell'Onu nel ig57. Parallelamente, Marc André analizza in diacronia lo statuto di cittadinanza delle Algerine (I93o-I96o), fino alla "cittadinanza variabile", la doppia nazionalità con doppio 
diritto di voto. Una cittadinanza sociale fondata su una corretta educazione di genere è nel programma di recupero dello stato del Messico, oggetto del successivo studio di Chiara Calzolaio, sulle vittime della criminalità organizzata dal 2006.

Nella sezione Regards complementaires, Diego Paiaro considera l'azione dei tirannicidi di Atene rafforzata dal legame omoerotico, a prima vista non coerente con il modello greco di cittadino, maschio e virile nella politica e nella guerra. L'eros è propedeutico all'esercizio della philia politica e non contraddice gli onori tributati dalla città per la andragathia: uniti contro il tiranno, la loro "cittadinanza ideale" risulta anzi rafforzata. Trasversalmente, l'azione di Madame Legros, benefattrice per la nazione francese nel xviı secolo, viene riletta da Laurence Croq come esemplare del passaggio dell'azione femminile dall'ambito familiare e privato all'ambito pubblico.

Nella sezione Documents, Philippe Akar studia i programata di Pompei, iscrizioni elettorali sui muri delle abitazioni nelle strade più frequentate per le attività commerciali, là dove gli elettori potenziali si affollavano. In una sessantina di iscrizioni una o più donne rogatores sostengono i candidati, affiancate o meno da cittadini maschi. La tradizione insiste sull'esclusione femminile dalla sfera pubblica, ma queste Pompeiane prendono pubblicamente la parola schierandosi per potenziali politici locali e chiedendo per loro il voto alla comunità. La presenza di nomi femminili stranieri e greci pone interrogativi sull'ampiezza della reale partecipazione femminile a situazioni pubbliche di interesse comune. Oggetto del successivo articolo, di Sara Panata, è la documentazione dell'attività del Women Movement of Nigeria, portavoce del malcontento femminile per conseguire il suffragio universale su base non censitaria, con una inclusione di ambito politico e sociale corrispondente alla nozione di cittadinanza nelle categorie di pensiero occidentali.
Per la sezione Actualité de la recherche, Violaine Sebillotte Cuchet propone un'importante disamina sulla definizione storiografica della cittadinanza femminile nel mondo ateniese e greco antico, con ricca bibliografia, rendendo conto del dibattito nella ricerca dall'ultimo ventennio del $\mathrm{xx}$ secolo a oggi. Nella città-stato greca l'esperienza del politico e delle pratiche del tà politikà - partecipare, condividere, decidere insieme - è stata per decenni considerata come esclusivamente maschile. A partire dagli studi di Nicole Loraux si è imposta per le donne greche la definizione di cittadine impossibili: se le donne possono essere nominalmente cittadine, esse restano categoricamente escluse dalla cittadinanza politica. Tale modulo storiografico è risultato rafforzato dagli studi di storia sociale di Pierre Vidal-Naquet sulla marginalità di schiavi e donne nella città greca e dalla sua celebre definizione della polis come club d' hommes. In realtà il fenomeno della cittadinanza antica è complesso e non può essere appiattito sulla politeia maschile. Indagini recenti hanno assegnato visibilità alla partecipazione femminile in altri campi pubblici di attività, nelle pratiche religiose e negli scambi economici, incrinando la validità della separazione privato-pubblico o interno-esterno. Il ruolo eminente di numerose donne nella sfera religiosa, per incarichi ufficiali, può considerarsi esemplare di una divisione delle attività secondo il genere; in questo contesto esse sono interamente cittadine. Tra II e I sec. a.C., nello stato ellenistico-romano, la documentazione diviene più copiosa e mostra la partecipazione delle donne ad attività pubbliche pro civitate.

Per la sezione Portrait, Leïla Sebbar propone un testo a carattere biografico, con figure femminili differentemente caratterizzate, in ambiente coloniale. Nella sezione Varia, Monica Bolufer Peruga rilegge la Défense des femmes (I726) di Benito Jerònimo Feijoo, valorizzandone l'attualità; a conclusione, un ampio studio di Ute 
Gerhard indaga sul rapporto controverso tra diritto e storia della condizione femminile nel xviı secolo.

Il volume Citoyennetés costituisce un contributo imprescindibile alle ricerche su cittadinanza e appartenenza femminili: tra mondo antico e realtà più attuali le questioni si richiamano trasversalmente per affinità e divergenze, incoraggiando il lettore a continue riflessioni.

Maria Luisa NAPOLITANo Università di Napoli Federico II napolitanoml@libero.it

Frédéric Colın, Olivier Huck,

Sylvie VAnseveren (éd.),

Interpretatio. Traduire l'altérité culturelle dans les civilisations de l'Antiquité, Paris, De Boccard, 20I5, 448 p., 59 euros / IsBn 978-2-70I8-0375-3.

L'étude des contacts culturels offre un champ de recherche très vaste. Aux thématiques actuelles d'acculturation (parfois controversée), de résiliences, de résistances, et de transferts culturels, il existe en matière de religion la notion appelée Interpretatio qui s'applique dans l'Antiquité principalement à des équivalences fonctionnelles et culturelles entre les dieux de différents panthéons. L'enjeu du recueil d'articles intitulé “Interpretatio. Traduire l'altérité culturelle dans les civilisations de l'Antiquité » consiste à étendre l'application de l'Interpretatio aux valeurs culturelles, linguistiques, iconographiques, normatives et diachroniques (avant-propos du coordinateur F. Colin, p. 8-12). Le but est ainsi de concevoir les transferts du point de vue du receveur et d'identifier les enjeux culturels liés aux emprunts, notamment dans la vie quotidienne. Lors de plusieurs séminaires, réalisés sous l'impulsion de l'Université de Strasbourg dans un cadre interdisciplinaire et européen, treize savants ont appliqué la notion d'Interpretatio à leur recherche.

La linguistique est le champ d'étude le plus représenté dans cet ouvrage. Si le fonctionnement des emprunts linguistiques et de leurs dérivés est bien connu par ailleurs, les études se concentrent ici sur l'incidence culturelle de ces interférences. Les limites de ces recherches pour les langues mortes sont rappelées par S. Venséveren (p. 15-34): deux facteurs certains à l'origine de l'emprunt sont la nouveauté et le prestige d'une langue. Un exemple de la supériorité du grec sur le latin se lit dans l'hellénisation de la colonie romaine de Philippes, étudiée par C. Brélaz (p. 37I405) : la mixité de provenance des habitants, la variété des langues maternelles, la non linéarité de l'utilisation du grec montrent que les colonies romaines n'ont pas vraiment été, au moins dans ce cas, des foyers actifs de romanisation des provinces et que les transcriptions en grec de mots et de noms étrangers sont également aléatoires.

Dans le champ de la traduction, la notion d'Interpretatio ou la recherche d'une équivalence semble nettement adaptée car, en l'absence de la notion correspondante, trois choix se présentent: l'Interpretatio (chercher un mot équivalent), la périphrase, la transcription (constatation de F. Colin, p. 35-64). Pour traduire des réalités politiques, D. Lenfant (p. 95-I22) propose l'exemple de la titulature des satrapes du roi Perse. Trois manières différentes se déclinent en fonction de choix culturels précis: l'Interpretatio par l'équivalence avec le mot Roi (basileus); la transcription phonétique comme “karanos » (le généralissime), “ azabarite », ou "satrape» avec beaucoup de variantes dans l'orthographe; ou un néologisme, qui est aussi une métaphore et non un titre, “l'œil du roi » voire “les oreilles du roi ». Chaque solution a des effets sémantiques précis qui insistent sur l'altérité, l'exotisme, ou souhaite gommer les différences. Pour une autre réalité méconnue, non plus 
sur le plan linguistique mais sur le plan civilisationnel, A. Jacquemin (p. I47-I6o) prend le cas des «barbares occidentaux». Les artifices littéraires pour rendre compte au lecteur hellénophone d'une réalité étrangère se fondent sur une différence de chronologie (les présenter comme vivant à la période d'Homère), sur les descriptions d'Hérodote pour l'Égypte et l'Orient, ou sur un vocabulaire étranger. A. Chauvot (p. I9I-2I2) étudie le choix culturel de la traduction du mot libyque “MNKD», tantôt par “imperator » tantôt par « roi », d'après les sources gréco-latines sur la rébellion de Firmus, fils de Nubel, en Maurétanie césarienne (372-375 ap. J.-C). L'équivalence et la traduction dépendent d'une présentation du chef de clan comme un usurpateur ou comme un descendant de chef. L'image est aussi ambivalente, entre le torque et la pourpre du manteau. Ces trois exemples montrent bien que, selon les choix de la traduction, des réalités culturelles côtoient des implications idéologiques et peuvent transformer complètement la vision de l'Autre.

Dans le champ iconographique, F. Labrique (p. 23I-264) présente la tunique historiée de Saqqara sur laquelle MaâtAlêtheia est présentée en Isis-Perséphone. Il doit s'agir d'un vêtement liturgique avec un programme iconographique qui associe des éléments grecs dans un décor égyptien comme notamment des objets (la massue d'Héraclès), la tradition grecque de la lune en lien avec Perséphone, et la profusion des aspects solaires en lien avec Apollon. Pour la divinité Artémis d'après O. Henri (p. 123-145), sa présence en Égypte correspond à l'équivalence avec Bastet/Boubastis ou Ouadjet leoncéphale ou Aat la lionne; ces différences dépendent d'une interprétation grecque locale, soit par rapport à une fonction de la divinité, comme son rôle protecteur dans la maternité, soit par rapport à un rapprochement iconographique. L'Autre est alors perçu iconographiquement dans une identification fonctionnelle qui le rend familier.

D'un point de vue juridique, les traductions de contrats ou de traités d'alliance donnent de beaux exemples de différences culturelles dans le choix des interprétations. A. Mouton et C. Van den Hoven (p. 67-93) montrent la variation des traductions de la liste des témoins divins du traité entre le roi hittite Huttušili 3 et le pharaon Ramsès 2. Il existe des traductions égyptiennes réalisées phonétiquement mais aussi des transcriptions qui comportent des erreurs, comme la confusion entre le nom d'un dieu et le nom d'une ville, et des équivalences comme le dieu de l'orage associé au dieu égyptien Souteckh. A. Delattre (p. 2I3-228) présente les variations de traduction entre le copte et le grec dans les monastères égyptiens (VII - -VIII ${ }^{\mathrm{e}} \mathrm{s}$.) : il note jusqu'à cinq mots différents pour désigner le supérieur du monastère de Baouit et des périphrases pour transcrire directement des notions grecques dans la langue copte. Dans le cadre d'une législation, O. Huck (p. 267-3ı5) étudie l'intégration, au $\mathrm{IV}^{\mathrm{e}}$ siècle, dans le droit de l'empire romain, des procédures de recours à la justice épiscopale qui existent dans la communauté chrétienne depuis le I ${ }^{\text {er }}$ siècle. Il constate quatre possibilités d'intégrer cette justice dans la législation: celles du tribunal juif et des procédures alternatives qui correspondent peu au phénomène chrétien; celles de la compétence de l'arbitre ex compromisso ou de la iuridictio d'un juge étatique, qui sont successivement utilisées dans le temps et qui provoquent de violentes querelles. Enfin, F. Colin (p. I6I-I9o) propose de voir sur des ostraka les jeux de variantes à partir d'une même sentence juridique pour traduire le verbe antikategoreis, qui correspond à une tactique de défense offensive pour éviter d'être accusé : il s'agit sûrement d'un essai de traduction par un élève et du corrigé par son maître, en rapport avec des livres d'exercices et une 
indigénisation des formules grecques. Les méconnaissances culturelles d'un monde à l'autre apparaissent dans les notions juridiques : l'adaptation est très difficile et donne lieu souvent à des contre-sens ou des interprétations très variées. Ces données permettent d'étudier autant le rapport entre deux cultures que d'approfondir le sens des enjeux linguistiques et culturels des civilisations réceptives.

La dernière partie concernant les rencontres culturelles diachroniques débute par une étude de N. Brout (p. 3I9-352) sur l'appréhension à la Renaissance des notions de la rhétorique antique, associée à des enjeux idéologiques. En effet, la reprise du latin et des textes de Cicéron permet de placer son utilisateur dans la querelle des Anciens et des Modernes et de critiquer la religion catholique par comparaison avec l'Antiquité. Plus près de nous chronologiquement, A. D'Hautcourt (p.355-368) étudie comment le monde latin est traduit dans le monde japonais à partir du manga Thermae Romae paru depuis 2008. La langue japonaise est ouverte aux emprunts lexicaux mais le latin se présente de manière phonétique ou par des néologismes ou par des phrases en latin non traduites. Ainsi l'image d'un Japon ouvert à l'altérité et pacifique est mise en avant par ce manga, ce qui est à l'origine du succès de la série. Dans ces Interpretationes diachroniques, le monde moderne se dévoile davantage : les enjeux idéologiques se lisent à l'aune du choix interprétatif. La manière de présenter l'Autre ou le Passé éclaire les attitudes du Présent et facilite la compréhension du monde contemporain.

Claire JONCHERAY UMR ArScAn claire.joncheray@free.fr
Angus Fletcher,

Comic Democracies. From Ancient Athens

to the American Republic,

Baltimore, John Hopkins University

Press, 2016, 224 p.,

49, 95 dollars / ISBN 978-I-42I4-I934-3.

L'idée principale du livre d'A. Fletcher est d'examiner la relation originelle entre la comédie antique et la démocratie athénienne pour voir, dans une démarche historique, comment cette relation fut récupérée, par divers genres littéraires et rhétoriques empruntant à la comédie, et à des époques plus récentes de la démocratie moderne, afin de nourrir cette dernière, de l'étayer ou de lui permettre de surmonter des crises. Cet examen de diverses époques amène Fletcher à faire l'hypothèse que certaines caractéristiques communes des “ démocraties comiques », comme il appelle ces représentations, peuvent aujourd'hui encore nous permettre d'améliorer nos pratiques démocratiques. L'ouvrage, en plus d'une enquête extrêmement riche sur une série de questions culturelles et politiques, est donc également un manifeste politique empreint de pragmatisme et riche d'optimisme.

Dans son introduction, Fletcher souligne que la démocratie ancienne, née avant tout de pratiques non théorisées, et la démocratie moderne, née avec la philosophie des Lumières, découlent de logiques différentes, sinon étrangères, mais partagent un principe commun essentiel, le pluralisme comme opportunité d'enrichissement. Il précise ensuite ce qui est pour lui la difficulté des démocraties les plus récentes, celles qui se maintinrent ou naquirent après la deuxième guerre mondiale : elles gardèrent une trop grande confiance en l'idéologie des Lumières, et ainsi eurent peine à s'adapter à des modèles nouveaux, comme celui du libre-échange. Or la comédie ancienne permet de réfléchir, par un décentrement historique, aux capacités d'adaptation profondes de la démocratie 
en général, par trois éléments qui sont à la fois caractéristiques de ses intrigues et de ses héros, mais aussi de la démocratie athénienne même : le pluralisme (avec la capacité à intégrer des éléments étrangers ou inédits dans le modèle civique), le pragmatisme et l'empirisme, trois principes, précisément, explorés par les nouveaux démocrates quand ils réfléchissent à l'adaptation nécessaire de leur modèle politique.

Afin de mener cette enquête, Fletcher consacre un premier chapitre à la comédie ancienne elle-même dans sa relation à la démocratie, et souligne qu'elle se présente comme un ensemble de méthodes et de réponses pratiques à des problèmes rencontrés par la cité, tels que la famine et la guerre en particulier. Ce caractère de méthode pratique, selon Fletcher, est ce qui relie essentiellement la comédie ancienne et la démocratie athénienne.

Dans les chapitres suivants, qui présentent cinq cas d'études, Fletcher se livre à un parcours historique dans lequel il étudie plusieurs formes comiques afin de montrer comment elles empruntent, plus ou moins directement, à la comédie antique, afin de développer ou d'améliorer des attitudes démocratiques. L'idée qui se précise dans ces chapitres est que cette amélioration est liée à un type d'humour inclusif qui permet, sans agresser les adversaires politiques puisqu'il se fonde en partie sur l'autodérision, de faire évoluer le modèle politique dans une démarche non pas utopique, mais pragmatique, et allant généralement vers une extension pluraliste du corps civique.

Le premier cas est celui de Machiavel, et des relations qui unissent Le Prince à ses lectures et ses expérimentations comiques : dans chaque cas se dessine la figure de l'impétueux, ou de l'audacieux, en comédie l'esclave par excellence, comme figure de progrès, d'adaptation et d'élargissement politiques. Le cas suivant est celui de l'Henry $V$ de Shakespeare, qui, selon Fletcher, et si l'on s'intéresse aux accents comiques de la pièce, est moins une représentation de la tyrannie qu'une mise en scène de l'imitatio comme vertu politique permettant au peuple de s'adapter et d'évoluer vers une forme de prise de pouvoir. Fletcher retrace ce principe d'imitatio jusqu'aux rhéteurs anciens, et en étudie l'influence après la pièce de Shakespeare. Le troisième cas (chapitre 4) est la reprise par Jefferson, dans son Préambule à la Déclaration d'Indépendance en $\mathrm{I} 776$, du principe d'indolence, qui remonte à l'ataraxie épicurienne, elle-même ensuite relayée par la comédie de Ménandre, puis par toute une tradition anglaise d'auteurs dramatiques comiques des XVII $-\mathrm{XVIII}{ }^{\mathrm{e}} \mathrm{s}$. L'idée commune de l'une à l'autre étape est que le bonheur est plus fondamental que la propriété, mieux, que cette dernière conduit en réalité à la misère. Le chapitre suivant étudie les relations entre les Pères Fondateurs de la démocratie américaine, l'Histoire du Déclin et de la Chute de l'empire romain d'E. Gibbon publiée en 1776 , et le type de narration employée par Cervantès dans la deuxième partie de son Don Quichotte, différents jalons les reliant. Les emprunts de l'un à l'autre sont en réalité ceux d'une méthode comique qui permet d'asseoir un gouvernement populaire et d'en éviter une dérive dangereuse, celle consistant à promouvoir un gouvernement de foule trop débridé, et qui risquerait de brimer les minorités essentielles au bon fonctionnement de la démocratie. Enfin, au chapitre 6, Fletcher montre comment ce type de méthode comique est également remployé par W. Irving et F. Douglass afin d'amender la Constitution, non plus en la refusant en bloc, dans la recherche utopique d'une perfection inaccessible, mais en s'efforçant de la faire évoluer, grâce à une argumentation nourrie d'humour inclusif, dans le sens de l'abolitionnisme. Cet humour inclusif, qui permet à un système collectif d'accueillir ce qu'il pense d'abord être l'imperfection, Fletcher le fait remonter à Homère et Aristophane. 
Dans un dernier chapitre, Fletcher étudie des cas plus récents de pièces postcoloniales, pour montrer comment cette remédiation comique peut encore répondre aux questions politiques contemporaines.

Anne de CrÉMoux Université de Lille anne.decremoux@univ-lille3.fr

David Hernández de la Fuente,

El despertar del alma. Dioniso y Ariadna: mito y misterio,

Barcelona, Ariel, 20I7, 423 p., 23,9o euros/ IsBN 978-84-344-2583-5.

El encuentro de Baco y Ariadna plasmado en el cuadro de Tiziano que actualmente expone la londinense National Gallery, destaca como punto de partida de este ensayo que, con magnífico bagaje de erudición, escritura delicada y orden modélico, procura un enfoque de amplio espectro del devenir de esta pareja mítica en la tradición literaria, iconográfica y operística de Occidente: «... seguramente, nadie con anterioridad había sabido captar como Tiziano la fuerza de ese momento de reconocimiento entre Baco y Ariadna [...]; el despertar de la joven cretense a la nueva vida que le procuraba el dios más fascinante de la Antigüedad y el que sin duda ha tenido un Nachleben más vivaz en la historia de nuestra cultura» (p. 25).

Elúltimolibro deHernándezdelaFuente ${ }^{1}$, profundiza en la complejidad de Dioniso consignando como constante referencial cel mitema del despertar de Ariadna». Con tal

1 Autor, entre otros monográficos de Oráculos griegos (Madrid, Alianza Editorial, 2008), Vidas de Pitágoras (Girona, Atalanta, 2011) y Mitología clásica (Madrid, Alianza Editorial, 2015). fin, el autor considera, claro está, estudios tan determinantes como los de Walter F. Otto (ig33), Henri Jeanmaire (ig5i), Walter Burkert (ı972), Károly Kerényi (I976) o Marcel Detienne (I977) ${ }^{2}$, desarrollando un cuadro cronológico, instruido e instructivo, de las más señaladas corrientes de aproximación a Dioniso, «el dios que marcará para siempre la modernidad"s.

Desde este particular acercamiento al más polifacético de los dioses griegos, se entiende el énfasis del autor en el punto de inflexión experimentado por el dionisismo en la fin de siècle europea, con la atención prestada al fenómeno del menadismo por Adolf Rapp (I872) y la norteamericana Elisabeth Robins (I883), con el acercamiento de Dioniso al universo contemporáneo procurado por Walter Pater (I876)... y, claro está, con la interpretación clave de lo dionisíaco planteada por Friedrich Nietzsche. Sin obviar el indudable giro que El nacimiento de la tragedia (1872) supuso para la recepción moderna de la temática en cuestión, el autor acierta a subrayar la deuda de esta obra para con reflexiones predecesoras, como la “dionisiología en Schelling» o la idea de voluntad desarrollada por Arthur Schopenhauer: “Hay una línea sutil pero inconfundible que transita desde la voluntad de Schopenhauer a las ideas sobre los patrones y estratos de la mente de Freud y Jung pasando de forma ineludible por el Dioniso nietzscheano» (p. 320).

2 También recogidas, entre otros monográficos en los, relativamente recientes, de Richard Seaford (Dionysos, LondonNew York, Routledge, 2006) o, en el ámbito español, de Diego Mariño Sánchez (Injertando a Dioniso. Las interpretaciones del dios, de nuestros días a la Antigüedad, Madrid, Siglo XXI, 2014), quien presta especial atención al «Dioniso contracultural» de la posmodernidad. 
Digna de resaltar es también la consideración del cambio de tendencia marcado por el estudio de los valores emocionales de la religión que, ya en el siglo XX, llevó a cabo Jane Harrison ${ }^{3}$ - enfoque, dicho sea de paso, que fue determinante para la composición del exitoso ensayo de Eric R. Dodds, The Greeks and the Irrational (I95I) -, así como la atención prestada a los estudios sociológicos sobre «la mujer dionisíaca» que afloran desde mediados del siglo XX (p. 333-337).

Pero nos demoramos aquí en los aderezos finales de un retablo que se ha consolidado atendiendo a las múltiples facetas que la mitología griega presta a Dioniso y a los primeros testimonios escritos del nombre "Ariadna" - de controvertida etimología (p. 120 ss.)- en las tablillas minoicas y micénicas, en los textos homéricos, en Hesíodo y Plutarco, así como a las variantes del mismo que presentan las inscripciones en la cerámica griega. Un retablo en el que El lamento de Ariadna de Nietzsche se contempla desde la "filosofía dionisiaca», cuyo inicio remonta al tema platónico de la Edad de Oro, para dar pormenorizada cuenta de la cristianización paulatina -al hilo de “la operación de ritualizar la filosofía que realizaron los neoplatónicos» (p. 20I ss.)de esta pareja divina, que encarna, al fin, la gozosa resurrección tras la muerte.

La interpretación del “despertar de Ariadna» como "katábasis al mundo de los muertos y regreso posterior al de los vivos mediante una resurrección que termina con la unión extática con la divinidad» (p. I94 ss.), destaca como punto álgido de un recorrido atravesado musicalmente por la ópera Ariadne auf Naxos (Ig12), cuyos compositores, Hugo von Hofmannsthal y Richard Strauss, perfilan el mito de la princesa cretense “como epítome de la

3 En especial, Themis. A Study in the Social Origins of Greek Religion, Cambridge, 1912. tensión entre lo antiguo y lo moderno, lo trágico y lo cómico, la muerte y la vida» (p. $357 \mathrm{ss}$.).

Este amplio estudio, concebido por su autor para un público variado, aclara temáticas esenciales para la comprensión del pensamiento moderno desde el estudio de la Antigüedad, al tiempo que evoca cuestiones que activan la imaginación del lector, dejándolo expectante ante la misteriosa "señora del laberinto» que pudo ser Ariadna en sus orígenes; ante los posibles rasgos semíticos de la devota entre todas las devotas de Dioniso; ante la eventualidad de que la Atenas impulsora del mito de Teseo la considerara como algo más que una princesa bárbara de la que el “fundador del sinecismo» debía liberarse, al igual que liberó a su polis de las, también resueltas, Amazonas; o ante la duda de si, en el pensamiento de la cristiandad, cupo que el alma revistiera un género específicamente femenino, más allá de la “anecdótica» simbología pagana.

El despertar del alma traza, en definitiva, un recorrido auténticamente iniciático en cuanto a la trascendencia salvífica -redentora- aprehendida a través de los siglos por el, al fin y al cabo, «misterioso» Dioniso y su redimida esposa.

$$
\begin{array}{r}
\text { Ana Iriarte } \\
\text { Universidad del País Vasco. España } \\
\text { ana.iriarte@ehu.eus }
\end{array}
$$

Ted Kaizer (éd.),

Religion, Society and Culture

at Dura-Europos,

Cambridge University Press,

“ Yale Classical Studies » 38, 2or6, 3ıо p.,

64.99 livres / ISBN 978-I-IO7-I2379-3.

Gli Atti del Congresso su Dura-Europos, tenutosi a Durham il I9-20 dicembre 2008, vengono pubblicati da T. Kaizer con alcuni 
aggiustamenti nell'organigramma e variazioni nei temi trattati.

A partire dall'Editor, quasi tutti gli autori dei saggi - L. Gregoratti, J.A. Baird, M. Sommer, L. Dirven, M.K. Heyn, J.-B. Yon, J. Buchmann, T. Gnoli, C.M. Acqua, J. Austin, L.T. Stuckenbruck, K. Ruffing, S.B. Downey, L.R. Brody - sono profondi conoscitori dell'universo culturale della regione di "frontiera" collocata tra il $34^{\circ}$ e il $35^{\circ}$ parallelo e tagliata dal corso del Medio Eufrate: la ben nota "fascia grigia" che vide la formazione e la compresenza di multiformi identità e di variegati intrecci di rapporti di carattere politico, etnico, religioso, artistico e linguistico. Qui, in tempi, modi e facies differenti, fiorirono in età ellenisticoparthico-romana realtà tra loro diversissime come Palmira, Dura-Europos e Hatra, nel cui odierno destino di distruzione/saccheggio/abbandono il grande Rostovtzeff, genius loci di Dura, certo constaterebbe una ciclica riproposizione degli eventi consumatisi nei decenni centrali del III secolo d.C.

In particolare, Dura-Europos, nelle tre fasi (greco-macedone, parthica e romana) che si succedettero nel mezzo millennio della sua esistenza (dal 3oo ca. (?) al 256 ca. d.C.), rappresentò un unicum come crogiolo formativo dei più vari fenomeni di "acculturation, hybridity, créolité" (cfr. Sommer), culminanti nella composizione di nuove - e per molti versi originali e mai definitive - sintesi.

Già agli artefici del disseppellimento della città - in primis ovviamente a Cumont e a Rostovtzeff (cfr. Kaizer) - si pose come prioritaria l'esigenza dell'individuazione delle origini e della morfologia degli elementi costitutivi ("mediterranei", ellenistici, siriaci, palmireni, "mesopotamici", iranici etc.) della cultura durena. Il problema fu riproposto in séguito alle campagne franco-siriane del I986-20II, tra le cui finalità (oltre a nuovi scavi e al restauro e al consolidamento di strutture già note) figurava anche un ripensamento critico delle precedenti ricostruzioni interpretative con l'utilizzazione di moderni strumenti e di nuovi modelli ermeneutici.

Molti saggi del pregevole volume curato da Kaizer muovono lungo questo percorso di ripensamento, che si configura ora come denuncia (cfr. Downey) dei macroscopici abbagli esegetici degli studiosi del passato, ora come ripresa di affermate teorie (cfr. Ruffing sul commercio "a medio raggio" - non carovaniero! - di Dura), ora come utile indagine su aspetti meno noti della documentazione (cfr. Baird, Austin), e infine come riformulazione, su singoli monumenti o affreschi, di ipotesi originali (cfr. Downey, Gnoli), talvolta non prive di audacia (cfr. Heyn).

In misura maggiore o minore, la revisione interpretativa presente nel volume investe aspetti del polimorfismo identitario delle varie componenti della società durena.

Con uno "sguardo da Oriente", Gregoratti congettura intorno alla stabilizzazione dell'oligarchia al potere in Dura, attribuendola a un disegno politico arsacide, mentre Gnoli, in controtendenza rispetto alla attuale communis opinio, ravvisa nel Mitreo di Dura elementi culturali di provenienza iranica. Con (prevedibile) "sguardo da Occidente" L. Dirven rivisita il problema "rostovtzeviano" della cd. "arte parthica", sostenendo che a Palmira - e non alla Mesopotamia arsacide - vanno riportati modelli e matrici dell'arte durena. Il rapporto con Palmira e, nel contempo, la specificità di Dura è osservata anche da L.T. Stuckenbruck nelle iscrizioni bilingui greco-palmirene delle due città.

Alla dinamiche legate alla subordinata posizione giuridica della donna e alla presenza femminile nei templi dureni riservano la loro attenzione Yon e Sommer, che esamina anche il caso della comunità ebraica residente a Dura, coinvolta in una dinamica di separatezza/integrazione. Lo stesso Yon e Buchmann indagano - sulla base della documentazione archeologica ed epigrafica - sulla funzionalità (e sulla polifunzionalità) delle "salles à gradins" e 
delle "sale da banchetto" presenti in molte aree templari della città.

Chiudendo questa telegrafica scheda di segnalazione, ricordo che il volume curato da Kaizer sottende oggi per noi un invito e un monito: le devastazioni e i saccheggi successivi all'interruzione dell'attività "sul campo" a Dura (primavera 20II) devono e dovranno essere in qualche misura compensati dall'assidua prosecuzione di studi e riflessioni su quanto ci è stato restituito dalla straordinaria "Pompei del deserto". Ne offre un esempio (e uno strumento) l'indefessa opera di conservazione, restauro, catalogazione e divulgazione promossa dalla "rostovtzeviana" Università di Yale (cfr. Brody).

Pier Giuseppe Michelotto Università degli Studi di Milano piergiuseppe.michelotto@unimi.it

Jacques Le Goff, Jean-Pierre Vernant, Dialogue sur l'histoire.

Entretiens avec EmmanuelLaurentin,

Bayard, Montrouge, 20I4, 92 p.,

I3 euros / IsBN 9782227487697.

Par ce livre, paru après la mort de ses deux protagonistes, le journaliste Emmanuel Laurentin offre le témoignage d'une série de conversations avec Jean-Pierre Vernant (I9I4-2006) et Jacques Le Goff (I924-2014), diffusées en janvier 2004 par la chaîne radiophonique France culture. L'importance d'une telle publication est proportionnelle à son statut de document : la forme des entretiens croisés permet de comparer la biographie et l'œuvre de JPV à celles de JLG. Il devient possible par là de réfléchir à une question d'histoire de la culture: celle des rapports entre l'anthropologie historique de la Grèce ancienne et l'anthropologie historique de l'Occident médiéval.
Des entretiens ressort la conscience, partagée par JPV et JLG, d'avoir suivi deux parcours de recherche contemporains et indépendants l'un de l'autre, mais convergents quant à l'approche historicoanthropologique des faits de civilisation. La question des origines ressort du premier entretien (Les maîtres). Philosophe de formation, JPV isole deux influences croisées : celle d'Ignace Meyerson, fondateur de la psychologie historique, avec qui il partagea les années de la résistance à Toulouse et, à partir d'après-guerre, celle de Louis Gernet, helléniste de formation durkheimienne, à son tour ouvert à l'influence de Meyerson. Ces évocations permettent de faire le pont avec les reconstructions historiographiques qui identifient, dans les travaux de Gernet postérieurs à 1948 et dans les recueils d'essais de Vernant des années ig6o-1970(Les origines de la pensées grecque, I962; Mythe et Pensée, I965, Mythe et Société, I974), les jalons fondateurs d'une anthropologie de la Grèce ancienne spécifiquement historique (cf. R. Di Donato, Per un'antropologia storica del mondo antico, I990). Pour sa part, JLG, historien de formation, reconnaît ses maîtres dans deux historiens, membres du jury pour son agrégation en I95o: Fernand Braudel et Maurice Lombard. À ceux-ci, il ajoute l'influence déterminante de l'œuvre de Marc Bloch (1886-1944), historien et héros de la résistance. Or, on sait que la revue des Annales, que celui-ci fonda en I929 avec Lucien Febvre, marqua l'intégration des objets de la sociologie durkheimienne dans le cadre de l'histoire, ainsi que l'émancipation de la discipline historique d'une approche strictement événementielle. La cohérence de JLG avec de telles origines est confirmée par la suite de sa biographie intellectuelle: proche de la revue des Annales, dirigée par Braudel (maître de la longue durée) dans les années I95o-ı96o, il parvint par la suite à promouvoir l'interlocution entre histoire et ethnologie. D'où la fondation d'une anthropologie historique de l'Occident 
médiéval, dont les bases théoriques et le programme, redevables, entre autres, aux travaux de Mauss, se trouvent déjà énoncés dans la préface à Pour un autre Moyen Âge (I977).

Les convergences entre les profils de recherche deJPV et deJLGressortentà partir du deuxième entretien. Laurentin évoque la “levée des barrières disciplinaires " qui les mena, par des voies indépendantes, à un dialogue avec des figures de référence dans le panorama des sciences humaines françaises dès les années ig5o : Claude LéviStrauss et surtout Georges Dumézil. Or, tant JPV que JLG assument la possibilité d'une vérification historique comme le paramètre essentiel pour évaluer forces et faiblesses des travaux du fondateur de l'anthropologie structurale d'une part et du théoricien de l'idéologie trifonctionnelle de l'autre.

L'adhésion à la dimension historique de la recherche ressort aussi du troisième et du quatrième entretiens (Travail et technique; Tragédie, liturgie). Pour des raisons biographiques différentes, la question de la variabilité de la fonction sociale et psychologique du travail selon les temps et les espaces s'est avérée cruciale, pendant les années I95o, pour le début des études tant de JPV que de JLG. Sollicité par Laurentin, JPV résume le sens des essais sur le travail réunis en Mythe et Pensée, nés d'une tentative de conciliation entre marxisme critique et psychologie historique (cf. R. Di Donato, Per un'antropologia storica, p. 2IO-2II) : une telle fonction s'avère n'être pas vraiment opératoire dans le monde grec ancien, sinon sous la forme d'une habileté technique très dévalorisée. Les résultats évoqués par JLG apparaissent symétriques et complémentaires: issues d'un intérêt pour l'histoire des idées, ses premières recherches sur les marchands et les intellectuels au Moyen Âge (publiées en volume en I956-I957) lui permettaient de saisir la préhistoire d'une idée positive de travail qui lui était contemporaine. La conscience de la différence irréductible des terrains d'études ressort du quatrième entretien : central pour la compréhension de la Grèce ancienne, ce fait social total qu'est le théâtre (phénomène à la fois religieux, politique et artistique) ne peut pas être détecté au Moyen Âge.

Dans le dernier entretien, Laurentin pousse les deux savants à tirer un bilan sur l'état des chemins de recherche qu'ils ont frayés, ainsi qu'à se projeter vers l'avenir. L'évocation par Vernant du centre Gernet (aujourd'hui ANHima) et du Groupe d'Anthropologie Historique de l'Occident Médiéval par JLG est accompagnée d'une évaluation positive des nouvelles études sur le statut des images, dont JPV lui-même a été le pionnier.

Le constat de tant de convergences à partir de deux parcours de recherche à première vue indépendants oblige à revenir à la question des rapports entre anthropologie du monde ancien et anthropologie de l'Occident médiéval. La question s'éclaircit à la lumière d'une intervention de R. Di Donato, dans le cadre du colloque parisien de 2015 sur l'anthropologie historique de JPV et de JLG, au titre éloquent: La force des ancêtres : Marc Bloch, Louis Gernet, Marcel Granet. Dès les années I980, Di Donato a pu identifier une relation d'échange entre Gernet, Bloch et le future sinologue Granet pendant leur séjour juvénile à la Fondation Thiers. Fortement influencé par Granet, dans les années 1930 Gernet collaborait à son tour avec les Annales de Bloch (Per un'antropologia storica, p. I5, 2I). Dans la préface à la réédition des Rois Thaumaturges en ig83, JLG (p. IV-V) citait les recherches de Di Donato au sujet de ces relations croisées. La convergence entre les deux anthropologies historiques s'explique donc par un processus génétique largement entrelacé. En I94I, Bloch et Mauss participaient notamment au colloque sur Travail et techniques organisé par Meyerson à Toulouse. Ce n'est pas un hasard si, à la fin du premier entretien, JPV (p. 28-29) évoque des racines communes à celles de JLG et 
des occasions, telles que les colloques de Meyerson, où “historiens, psychologues, anthropologues, hellénistes » pouvaient se retrouver.

Carlamaria LuCCI

Università di Pisa carlamarialucci@interfree.it

Justine Mac Connell, Edith HaLl, Ancient Greek Myth in World Fiction since 1989 ,

Londres-New York, Bloomsbury

Publishing, 20I6, 273 p., 9o livres / IsBn 978-I-4725-7938-6.

L'ouvrage résulte d'un colloque organisé en 2012 par E. Hall et K. Billotte. Les éditrices ont réuni quinze contributions portant sur des œuvres de toutes nationalités et couvrant une vingtaine d'années (de I989 à 2009). Le projet d'ensemble est annoncé dans l'introduction: il s'agit de réfléchir aux tendances suivant lesquelles on a repris les mythes grecs dans la fiction d'après I989 - autrement dit, la fin de la guerre froide, la chute de l'URSS, la montée d'une nouvelle ère postcoloniale et l'éclatement d'une série de conflits, comme ceux des Balkans ou de l'Irak.

Dans cette nouvelle configuration mondiale, marquée entre autres par la globalisation culturelle, les éditrices pensent en effet que l'on peut trouver une certaine cohérence, dans la façon dont les mythes sont repris et liés au folklore d'autres époques et lieux d'une part, et dans leur exploitation pour permettre un geste critique, souvent social et politique, sur le monde décrit d'autre part. C'est cette cohérence par-delà les frontières, qui, d'après elles, permet de parler de world fiction, dans la lignée de la Weltliteratur définie d'abord par Goethe.

Les éditrices signalent les précautions à prendre lorsqu'on utilise de telles notions et reconnaissent les critiques qu'elles peuvent susciter. Elles rappellent néanmoins l'enjeu politique et de dialogue culturel que ces questions représentent dans le monde d'aujourd'hui.

L'introduction se termine sur une riche bibliographie sur ce sujet.

Dans les essais qui suivent, les approches, les contours des œuvres étudiées et leurs contextes sont très disparates, si bien que la convergence annoncée en introduction n'apparaît pas toujours explicitement. En outre, les essais ne renvoient pas les uns aux autres lorsque des mises en relation seraient possibles (par exemple, les textes $\mathrm{n}^{\circ}$ I et 4, qui étudient le roman brésilien, font des observations semblables sur son évolution pendant et après la dictature). Mais chacun des articles a un réel intérêt en soi et l'ouvrage dans son ensemble est une mine pour appréhender la réception du mythe grec dans la fiction récente.

P. Rankine aborde le roman brésilien pendant la dictature militaire et après $\mathrm{I} 989$, en le replaçant dans l'histoire du Brésil au $\mathrm{XX}^{\mathrm{e}} \mathrm{s}$., et dans une continuité de références aux mythes grecs.

W. M. Hutchins se concentre sur un roman de l'écrivain d'origine libyenne I. Al-Koni, Les Mages (I989). Son originalité est qu'il combine le mythe et le folklore touaregs et arabes aux mythes de l'Égypte et de la Grèce anciennes.

S. Perris étudie deux romans néozélandais de W. Ihimaera, The Matriarch (I986) et The Dream Swimmer (I997) (non traduits en français). Le premier s'inspire de l'épopée comme somme d'expériences, tandis que The Dream Swimmer reprend la trame conceptuelle de l'Orestie, en lien, à chaque fois, avec l'histoire des Maori.

S. Frade aborde un roman brésilien publié en 1990, Videiras de Cristal, de L. A. de Assis Brasil (non traduit en français), qui porte sur l'histoire des Mucker dans le Brésil du XIX ${ }^{\mathrm{e}}$ s., et sur les enjeux liés alors à la culture classique. 
L. Hardwick étudie deux exemples de “transition literature », une littérature de crise qui émerge de contextes de changements, pour y déceler la place du mythe. Il s'agit de Médée de Ch. Wolf, et de Ways ofDying (non traduit en français) de Z. Mda.

G. Amitrano se concentre sur les références aux mythes grecs dans la littérature japonaise et, parmi plusieurs exemples, aborde l'œuvre de Murakami, en particulier Kafka sur le rivage (2002), inspiré d'Edipe Roi.

A. Ganz propose une étude de la série The Wire, fondée sur les déclarations de son créateur, D. Simon, à propos de son remploi de la tragédie et de l'Iliade. Cet héritage lui permet en particulier de dramatiser le conflit entre les forces de l'ordre et la culture de la drogue.

F. Macintosh aborde un roman canadien de Th. Kishkan, A man in a Distant Field (2004, non traduit en français) et montre comment le biais de l'Odyssée, via Joyce, permet d'y présenter une réflexion sur l'histoire de l'Irlande aux XIX ${ }^{\mathrm{e}}$ et XX ${ }^{\mathrm{e}} \mathrm{s}$.

A. Ljunggren étudie ensuite le roman russe Minotaure.com : Le Heaume d'horreur de V.Pelevine, paru en 2005. Dans cette œuvre expérimentale écrite sous la forme d'un chat, le mythe du Minotaure permet de réfléchir à la nature de la nouvelle communauté crée par internet.

S. Matzner aborde la manière dont B. Schlink, dans Le Retour, utilise la construction odysséenne pour mettre les relations familiales au centre de l'interrogation sur la construction de l'identité nationale allemande.

E. Hall propose une lecture des Bienveillantes de J. Littell en montrant la manière dont l'auteur y articule une recherche documentaire poussée sur l'holocauste au remploi de deux mythes en particulier, ceux d'Oreste et de Narcisse.

E. Spentzou montre ensuite comment, dans le roman grec Aidonopita, d'I. Zourgos, paru en 2008, la culture grecque classique est reprise pour exposer les mythes de colonisation qui entourent la Grèce moderne, et la question actuelle de l'identité grecque.

M. Reynolds livre une étude de deux romans australiens, Une rançon de D. Malouf (2009) et Grand homme de Ch. Hooper (2008), où l'épopée homérique est le détour par lequel les auteurs abordent la violence systémique envers les Aborigènes.

H. Eastman montre ensuite comment le début de ce millénaire a vu naître un nouveau type d'héroïne adolescente, inspirée d'Antigone, à travers deux exemples très différents, Une Antigone à Kandahar de J. Roy-Bhattacharya, et Hunger Games de S. Collins.

Enfin, J. Mac Connell étudie l'emploi de deux mythes dans Ce qu'on peut lire dans l'air de D. Mengestu, la Télémachie et l'Orestie, et comme ils concourent à représenter l'expérience de la migration et la quête de l'identité.

Anne de Crémoux Université de Lille anne.decremoux@univ-lille3.fr

Angelo Mazzocco, Marc Laureys (éd.), ANew Sense of the Past. The Scholarship of Biondo Flavio (I392-I463),

Leuven University Press, Leuven, 20I6, 288 p.,

59,5o euros / ISBN 9789462700482.

L'importance de ce recueil d'essais pour les études néo-latines peut être appréciée à la lumière de l'introduction d'A. Mazzocco (Mount Holyoke College) sur la vie et l'œuvre de Biondo Flavio (1392-I463). Né à Forlì (Romagne), il fut notaire et secrétaire à la cour papale de I/333 jusqu'à sa mort, sauf pour une interruption entre 1449 et I453. Proche du pape Eugène IV (I43I-I447), qu'il suivit au concile de Florence, il participa au débat humaniste sur les origines 
de la langue vulgaire en Italie, d'où naquit le traité De verbis Romanae locutionis (I435). Suivirent un ouvrage historiographique de longue haleine (Historiarum ab inclinatione Romani imperii decades), qu'il acheva en I453, une description des architectures de Rome ancienne (Roma instaurata, I446), et une description des régions d'Italie sur une base géographique et historique (Italia illustrata), composée entre I 448 et I 453 , puis remaniée. Il se consacra enfin à une description des institutions de Rome ancienne avec une ambition de totalité : Roma triumphans (I459). Ses ouvrages connurent des éditions imprimées dont la dernière fut pendant longtemps l'édition bâloise de 1559 (chez Froben). Apparemment marginalisée par la suite, la production littéraire de Biondo a connu un regain d'intérêt au xIX ${ }^{e}$ siècle et surtout au fil du xxe , grâce à des travaux tels ceux de Nogara, Dionisotti et Fubini. À partir des années 2000, la nouvelle vague d'intérêt a pris la forme tant de véritables éditions critiques, principalement par les soins de l'Istituto Storico Italiano per il Medioevo, que d'éditions équipées de traductions en langue française ou anglaise : dans ces entreprises sont impliqués les auteurs des contributions. Celles-ci se situent donc au niveau de la constitution du texte et/ou de l'interprétation.

F. della Schiava (Université de Bonn) rend compte de la recension effectuée sur tous les témoins disponibles de la Roma instaurata, à partir d'un travail préliminaire avec M. Laureys (professeur de la même université), en vue de sa prochaine édition ISIME, alors que J. White (St. Bonaventure University) dresse un bilan sur les phases rédactionnelles de l'Italia illustrata, en tenant compte des résultats des recherches de P. Pontari (Italia Illustrata, I, ISIME, 20II). Les deux contributions convergent sur un point : l'évidence mène à identifier, dans les variantes des premières éditions de la Roma instaurata et de l'Italia illustrata, procurées par Gaspare, fils de Biondo Flavio, respectivement en I47I et en I474, les traces d'une extrême révision rédactionnelle non du père mais du fils.

En ce qui concerne le niveau de l'interprétation, deux essais de Mazzocco (Some PhilologicalAspects of Biondo Flavio's Roma Triumphans, I979; Biondo Flavio and the Antiquarian Tradition, I985) avaient déjà souligné les relations étroites entre la Roma instaurata et la Roma triumphans en tant qu'œuvres pionnières dans la reconstruction des antiquités romaines, par l'exploitation conjointe des sources littéraires et archéologiques. À l'intérieur du recueil, Laureys explore les forces et les limites de la Roma instaurata à travers les notes de lecture du prince-évêque de Trente Johannes Hinderbach (I418-I/486), alors que F. Muecke (University of Sydney) suit la fortune changeante, souvent souterraine, de la Roma triumphans dans la tradition des commentaires érudits produits entre le $\mathrm{XVI}^{\mathrm{e}}$ et le $\mathrm{xvIII}^{\mathrm{e}}$ siècle.

Un acquis particulier du recueil réside dans la mise en évidence du lien qui court entre la Roma instaurata, la Roma triumphans et les autres ouvrages de Biondo. La question critique soulevée par A. Momigliano, concernant le statut des traités d'antiquités dans l'histoire de la culture (cf. R. Di Donato, Prefazione a A.M., Decimo contributo alla storia degli studi classici e del mondo antico, 2012, p.X), est évoquée par C. Castner (University of South Carolina) à propos de l'Italia illustrata: il s'agirait d'évaluer le rapport, plus ou moins achevé, qui s'y établit entre l'étude des sources et leur agencement dans une grille temporelle, afin d'en déterminer le poids pour la construction d'une méthode historiographique moderne (p. 192). Or, un tel questionnement s'avère sous-jacent à la plupart des essais et justifie le titre du recueil, résumant l'apport culturel de Biondo et de ses œuvres dans l'élaboration d'un nouveau sentiment du passé.

G. Marcellino (SNS, Pise) souligne l'importance de la notion de décadence (declinatio/inclinatio) dans le cadre d'une 
enquête sur les sources littéraires du De Verbis. Bien qu'elle soit thématisée seulement plus tard dans les Decades à propos de la chute de l'empire romain, celle-ci permet de mieux comprendre la connotation négative du mélange linguistique (mixta loquela) que Biondo présente comme le résultat de la transformation $\mathrm{du}$ latin induite par les invasions barbares (p. 47-48). F. Delle Donne (Università della Basilicata) observe, d'ailleurs, que la formulation explicite de la notion d'inclinatio Romani imperii correspond à un changement plus général du plan des Decades qui aurait eu lieu, d'après la correspondance de Biondo, entre I/443 et I/46, c'est-à-dire pendant l'époque de composition de la Roma instaurata. Le passage d'un projet d'histoire contemporaine à un projet plus vaste (couvrant les dix siècles compris entre la chute de l'empire et son époque), uni à un élargissement de la perspective, de l'Italie du nord à l'Italie entière, auraient donc été sollicités par l'effort de compréhension des vestiges romains et par l'exigence de jeter un pont entre ceux-ci et le présent (p. 69, 78-79, 84-85). Le schéma historiographique des Decades est reconnu par Pontari (Université de Pise) comme un arrière-plan indispensable à l'intelligence de l'Italia illustrata et de sa nouveauté : l'introduction d'une notion d'Italie comme réalité culturelle (l'Italia nova des communes), issue de la transformation irréversible de l'héritage romain à travers l'apport barbare (p. i6ı-I64). Pontari (p. I5II58) met aussi en évidence les limites d'un tel point de vue: d'une part, le schéma décadence-renaissance est aujourd'hui mis en discussion par certains historiens ; d'autre part, Biondo voyait dans la papauté l'héritière directe de l'empire romain, dans le cadre d'une opposition binaire entre les Chrétiens et les Turcs qui venaient de s'emparer de Constantinople (cf. Mazzocco, p. I4-I5; Delle Donne, p. 8I-84). Au niveau de la méthode, la non-conformité au modèle rhétorique cicéronien, relevant d'une attention prioritaire aux sources, fait en revanche des Decades une œuvre pionnière, en rupture avec l'historiographie de son époque (Mazzocco, p. IO2-Io3). Plus généralement, à travers la mise en évidence d'un réseau de correspondances internes à la production littéraire de Biondo, les différents essais permettent d'y détecter une cohérence essentielle: il s'agit d'une réflexion précoce sur le passé de l'Italie à partir d'une méditation sur les antiquités romaines et leurs ruines.

Carlamaria LuCCI
Università di Pisa
carlamarialucci@interfree.it

Seth L. Schein,

Homeric Epic and Its Reception.

Interpretative Essays, Oxford;

Oxford University Press, 20I6, 227 p.,

52 livres / IsBn 9780I995894I8.

Seth L. Schein propose un recueil d'essais, disponibles jusqu'alors uniquement aux États-Unis. L'auteur, qui a consacré une grande partie de sa carrière à l'interprétation des textes homériques, cherche également à dépasser la querelle divisant les spécialistes de la question homérique, entre Analystes et Unitaristes. L'auteur se place ainsi dans la lignée de H. Fränkel et M. Parry, mais sollicite aussi les théories de I. Kakridis. Les douze chapitres de l'ouvrage sont pour la plupart des transcriptions d'interventions orales, dont le style a parfois été préservé, et tous ont déjà été publiés sous des formes abrégées, à part les chapitres 7, 9 et II.

Le chapitre i propose une analogie entre la mort du jeune Troyen Simoeisios au chant IV de l'Iliade et les destins d'Hector et de Troie, mettant en scène un thème fondamental du poème: le coût humain de l'action héroïque. Le chapitre 2 analyse 
en sections la scène du combat autour du corps de Patrocle au chant XVII et montre comment le point focal du passage est déplacé vers les chevaux “humanisés " d'Achille. Le chapitre 3 est une prise de position de l'auteur par rapport à l'analyse donnée par D. Page en ig55 de la scène entre Ulysse et Polyphème au chant IX de l'Odyssée. Alors que Page proposait d'y voir les étapes d'une sorte de " thème légendaire ", Schein le réfute en plaçant au centre la question de l'identité. Le chapitre 4 analyse les différents usages de l'allusion mythologique dans les deux poèmes. D'après Schein, les allusions mythologiques de l'Iliade permettent d'évacuer des thèmes autres que celui porté principalement par le poème; au contraire, celles de l'Odyssée servent à intégrer des alternatives. Le chapitre 5 se concentre sur “ l'humanité » et la "divinité » en tant que thèmes poétiques dans l'Hymne homérique à Aphrodite et celui consacré à Déméter. L'auteur pense que la puissance des déesses y est “ réajustée » en fonction de la relation qu'elles entretiennent avec Zeus. Le chapitre 6 s'intéresse à deux exemples d'intertextualité entre l'Iliade et l'Odyssée. Le premier exemple - le duel entre Iros et Ulysse pour une panse de brebis dans l'Odyssée est comparé au duel entre Ménélas et Pâris pour Hélène dans l'Iliade - permet à l'auteur de montrer comment un événement est mentionné de manière quasi-indépendante dans l'Iliade alors qu'un élément du même type est traité différemment dans l'Odyssée qui préfère l'intégrer complètement à sa propre structure narrative. Quant au second exemple - le rejet “ achilléen » par Ulysse de la supplique d'Eurymaque au chant XXII de l'Odyssée - il questionne le contraste habituellement remarqué entre l'héroïsme d'Achille et celui d'Ulysse. Dans le chapitre 7, Schein prend le parti de l'approche neurolinguistique cognitive, aux dépens de la traditionnelle approche esthétique, pour l'analyse des poèmes homériques pris à la fois en tant que performances et textes littéraires. L'auteur affirme l'importance du defamiliarization principle, qui consiste à contrarier les attentes psychologiques du public et dont les limites en matière d'objectivité peuvent être dépassées par la neurolinguistique. Les chapitres 8 et 9 permettent d'y voir plus clair dans les travaux des deux grands théoriciens dont se réclame Schein: l'interprétation littéraire de Milman Parry et la Neoanalysis d'Ioannis Kakridis. Le chapitre 8 se veut une critique de l'approche présentée par le premier dans sa leçon de 1943 (The Historical Method in Literary Criticism). Le chapitre 9 aborde quant à lui la Neoanalysis de Kakridis qui émet l'hypothèse d'un vivier de récits folkloriques pré-homériques dans lequel le poète serait venu puiser. Le chapitre io propose une analyse du poème "Priam's Night-Journey » du poète grec Constantin Cavafis (I863-ıg33). Schein s'attache à identifier des procédés “callimachéens » dans le travail du poète et rapproche ainsi le travail de Cavafis d'un exercice d'interprétation. Pour son avantdernier chapitre, Schein s'intéresse aux conceptions de la guerre dans l'Iliade et à travers quatre œuvres de réception :L'Tliade ou le Poème de la Force de Simone Weil, On the Iliad de Rachel Bespaloff, Memorial: An Excavation of the Iliad d'Alice Oswald et WAR MUSIC de Christopher Logue. L'auteur s'interroge également sur les apports de ces réceptions de la guerre “iliadique» pour penser la guerre de nos jours. Enfin, le dernier chapitre apporte un éclairage particulièrement intéressant sur un phénomène inconnu en Europe, les great books courses américains. Schein propose d'abord un historique de ces cours, puis il s'intéresse aux traductions produites par ces enseignements, pour enfin montrer comment la lecture d'Homère qui y est faite, mue par une montée des "valeurs nationales », a pu influencer l'interprétation des poèmes homériques par le lectorat américain du $\mathrm{XX}^{\mathrm{e}}$ siècle. 
L'auteur précise que les bibliographies fournies à la fin de chaque chapitre déjà publié ont été mises à jour, mais on regrette l'absence d'un certain nombre d'ouvrages francophones - bien qu'Hélène Monsacré et Pierre Vidal-Naquet soient cités ainsi qu'un article de cinq pages de JeanPierre Vernant - qui auraient apporté aux réflexions déjà très riches de l'auteur (J.-P. Vernant pour le chapitre 4, V. PirenneDelforge et Pironti pour le chapitre 5, ou P. Payen pour le chapitre II). Enfin, on aurait apprécié un mot de conclusion, même si une véritable conclusion générale aurait été un exercice probablement artificiel, l'ouvrage se voulant un rassemblement d'essais variés autour du thème homérique et de sa réception. Même si on peut regretter l'arrivée tardive des outils théoriques de Parry et Kakridis, le lecteur observateur comprendra que l'organisation des essais, portant d'abord sur l'interprétation des textes homériques eux-mêmes - majoritairement de l'Iliade néanmoins - puis sur les approches et réceptions modernes de ces poèmes, permet d'ouvrir un peu le champ des études homériques.

Sandya Sistac

Université de Toulouse (UT2J) sandya.sistac@gmail.com

Arbogast Sснмітт,

Wie aufgeklärt ist die Vernunft der

Auklärung? Eine Kritik aus aristotelischer

Sicht,

Heidelberg, Universitätsverlag Winter, 2016, 472 p.,

42 euros, ISBN 978-3-8253-646I-8.

On n'en a jamais fini avec les Lumières - en allemand Aufklärung. En dehors du XVIII ${ }^{e}$ siècle, surtout des années I740 à la veille d'une Révolution qu'elles semblent avoir engendrée - dans des représentations familières aux Français, depuis le transfert au Panthéon des restes de Voltaire et de Rousseau en I79I et 1794, mais aussi, chez Hegel et les hégéliens, Robespierre comme “main de Rousseau»-, chaque époque semble revendiquer ses propres Lumières, sa face " éclairée » et " progressiste ", sa force intellectuelle de contestation de l'ordre établi, proposant sa réforme ou son bouleversement dans le sens supposé de la liberté et de la justice.

Il y aurait ainsi des Lumières dès la Renaissance, puis au XvII ${ }^{\mathrm{e}}$ siècle (Descartes, les cartésiens, les Libertins, Spinoza), et, au-delà du XVIII ${ }^{\mathrm{e}}$ siècle et du "splendide lever de soleil » (Hegel) de i789, celles du premier XIX $^{\mathrm{e}}$ siècle: le libéralisme européen de i83o et au-delà, le saint-simonisme, l'hégélianisme "de gauche » ou critique, après la mort du maître Hegel en i83ı, et le mouvement ouvrier et socialiste à ses débuts en Grande-Bretagne et en France, et ainsi de suite jusqu'à nos jours ou presque.

C'est dans ce “presque » que - comme le dit une expression allemande plus rare en français - " gît le lièvre ». On sait en effet que, à partir de la "guerre civile européenne» que fut la Première Guerre mondiale, puis après l'indépassable - du moins l'espèret-on - sortie de l'humanité (a-humanité, et non pas simplement inhumanité, d'où l'on peut toujours revenir, Krouchtchev, puis Gorbatchev « revenant » du stalinisme) représentée par le nazisme exterminateur, il devint de plus en plus difficile de maintenir en bon ordre de marche des Lumières sans cesse renaissantes.

On sait aussi qu'une critique de fond de ces Lumières peut-être illusoires fut formulée au milieu des années I940 par deux des théoriciens d'une École de Francfort imprégnée d'hégélianisme et de marxisme, alors en exil aux États-Unis, Adorno et Horkheimer, dans une Dialectique de la Raison (Dialektik der Aufklärung - « raison » traduisant ici Aufklärung) qui s'efforçait de montrer comment la raison critique avait, paradoxalement, ouvert la voie à un retour de «mythes » supposés irrationnels et aux 
plus atroces régressions et transgressions. Depuis, on a tenté de différentes façons de prouver, entreprise à la fois aisée et inaboutie, que les divers “ totalitarismes » du $\mathrm{XX}^{\mathrm{e}}$ siècle étaient embryonnairement présents dans la "volonté générale de Rousseau comme dans le radicalisme “jacobin» des années I790, parfois à tort identifié à un lénino-stalinisme avant la lettre.

C'est peut-être de tout cela qu'il s'agit, de façon un peu contournée, dans ce travail érudit de l'helléniste allemand Arbogast Schmitt. Il s'agit de montrer, à partir d'une connaissance précise du grand Aristote, que la raison (Vernunft, et non pas le modeste entendement quotidien, Verstand) occidentale de l'ère moderne - au sens large - ne saurait rendre compte adéquatement des phénomènes sociaux ou idéologiques (les religions en particulier) propres à d'autres “cultures » ou civilisations. L'assurance factice que procure la maîtrise de la raison conduirait à des difficultés accrues dans les rapports entre sphères culturelles ou civilisations différentes. La question n'est pas nouvelle et demeure perpétuellement en débat, y compris à propos de lourdes questions internationales qui sont l'actualité des médias.

La raison occidentale ne sort pas toute armée, telle Athéna, de la tête des Descartes, Kant, Hegel et Comte, voire Marx. Elle a, elle aussi, une genèse complexe, et d'autres sphères culturelles que la nôtre ont bien le droit de la considérer, en retour, comme un exotique objet d'étonnement et d'étude. Aristote avait, en son temps, insisté - il faut le rappeler, ce que l'auteur ne semble pas faire, du moins si le recenseur y a vu suffisamment clair dans les références accumulées - sur l'étonnement comme point de départ de la recherche de la connaissance en tous domaines, c'est-àdire de la philosophie.

Aristote, avec la longue durée de sa « réception » en Occident comme en Orient, avec des médiations complexes, apparaît ainsi comme l'instrument privilégié d'une rencontre vivante, aujourd'hui encore, à partir d'un point de vue en quelque sorte excentré, entre des “cultures » et civilisations différentes comme entre des époques différentes.

On saluera, pour conclure, la grande ambition intellectuelle de l'auteur et l'ampleur des perspectives qu'il dégage, mais on regrettera l'ensevelissement de l'essentiel, parfois, sous l'avalanche des détails, ensevelissement perceptible, dès le sommaire, dans les nombreuses subdivisions des nombreux (vingt-deux) chapitres. Marque d'un aristotélisme imitatif?

$$
\begin{array}{r}
\text { Lucien CAlviÉ } \\
\text { Université de Toulouse (UT2J) } \\
\text { claude.calvie@orange.fr }
\end{array}
$$

\author{
Richard Sorabu (éd.), \\ Aristotle Re-Interpreted. \\ New Findings on Seven Hundred Years of \\ the Ancient Commentators, \\ Bloomsbury, London-New York, 20I6, IX \\ +673 p., \\ I40 livres / ISBN 978-I-47259-656-7.
}

Aristotle Re-Interpreted è la seconda raccolta di studi sugli antichi commentari ad Aristotele curata da Richard Sorabji, e deve perciò essere considerata insieme ad Aristotle Transformed. The Ancient Commentators and their Influence (I990, seconda edizione London-New York, Bloomsbury, 20I6). Entrambi i volumi hanno lo scopo di offrire un ampio quadro della ricerca sui commentari aristotelici nell'antichità, grosso modo nel periodo che va dal primo secolo a.C. fino al sesto secolo d.C. (con qualche incursione oltre questa data). Nei due casi, abbiamo dei saggi che sono in parte originali, in parte ristampati, preceduti da una lunga e riassuntiva esposizione del curatore. Il fine di queste raccolte è doppio. Anzitutto, 
mettere a disposizione dei lettori lavori dispersi in sedi varie, selezionati all'interno di un campo eterogeneo e assai esteso dal punto di vista cronologico. Al tempo stesso, offrire un quadro più o meno coerente, o almeno più consistentemente articolato, dell'argomento che unifica i vari contributi: i commentari ad Aristotele dall'antichità fino al primo medioevo. I volumi sono direttamente collegati al progetto di traduzione in inglese delle opere degli antichi commentatori di Aristotele iniziato nel I987, di cui è sempre responsabile R. Sorabji (coadiuvato poi da M. Griffin). L'impresa, che conta ormai più di cento volumi, si pone sulla scia del grande lavoro di edizione dei Commentaria in Aristotelem Graeca, collezione curata da Hermann Diels tra il I882 e il Igog (Berlin, Reimer). I commentari tradotti in inglese non sono accompagnati dai testi originali, ma offrono al lettore specialista ampie introduzioni e note, permettendo un più facile accesso ad autori ed opere poco frequentati. A questi grandi lavori editoriali si possono infine aggiungere le tre utili raccolte di fonti edite sempre da Sorabji: la "filosofia dei commentatori", tra il terzo e il settimo secolo della nostra era (London, Duckworth, 2004), rispettivamente nel campo della psicologia (vol. I), della fisica (vol. 2) e della logica e metafisica (vol. 3).

L'ampiezza di una tale produzione sui commentari ad Aristotele corrisponde certamente alla vastità di un campo di studi dai confini imprecisi e dai contenuti poco omogenei. Il tema dei commentari aristotelici costringe gli studiosi a seguire un movimento centrifugo che finisce per includere, tra i testi studiati, buona parte della riflessione filosofica dell'antichità, a partire dal primo secolo a.C. L'autore antico di un commentario ad un testo aristotelico può, ad esempio, avere pubblicato scritti di altra natura e va, in questi casi, considerato nell'unità del suo pensiero. I commentari stessi non costituiscono un genere omogeneo, ma sono dotati di una storia propria che deve essere attentamente considerata. Nemmeno è costante il legame esclusivo con Aristotele in tutta questa produzione, e l'influsso del platonismo è, in certi periodi, preponderante (il cosiddetto "aristotelismo dei non aristotelici", per riprendere l'espressione di Paul Moraux). Il tema, inoltre, della trasmissione dei testi guida solo in parte l'attenzione degli studiosi di questa letteratura. Il fine sottinteso resta sempre quello di mettere in evidenza non soltanto la costituzione di una tradizione testuale e filosofica, ma il carattere originale della riflessione di autori che si esprimono filosoficamente attraverso l'esegesi dei testi aristotelici: il commentario è una delle principali forme di espressione filosofica durante questi secoli.

Se non si deve quindi pensare all'argomento di queste raccolte di saggi come qualcosa di unitario, tuttavia esse permettono di richiamare l'attenzione su una letteratura spesso trascurata, di difficile comprensione e il cui valore intrinsecamente filosofico deve essere illuminato grazie ad un'ardua impresa "meta interpretativa" - vale a dire, la lettura degli interpreti in quanto autori filosofici. Ne servono da esempio i vari commentari alle Categorie aristoteliche, oggetto di più studi raccolti in questo volume e banco di prova dei rapporti non solo conflittuali tra platonismo e aristotelismo.

Gli stessi confini temporali si mostrano assai fluidi: il saggio iniziale di M. Hatzmichali espone con chiarezza i limiti delle analisi tradizionali che valorizzano in modo eccessivo la riscoperta dei testi aristotelici nel primo secolo a.C., a partire dall'appropriazione sillana della biblioteca di Apellicone e l'edizione di Andronico. E la conclusione tradizionale (già in Diels) della sequenza dei commentari aristotelici antichi con Eustrazio e Michele d'Efeso non deve condurre ad assumerli come punto naturale di approdo di una tradizione esegetica dotata di una qualche coerenza evolutiva. 
I lettori di questi saggi non avranno soltanto da meditare, in modo più generale, sulla storia della produzione di commentari e sul carattere della riflessione filosofica nei primi secoli dell'era cristiana nel loro rapporto mutevole, ma mai del tutto secondario, con l'opera aristotelica. I ventitré contributi riuniti in questo volume (insieme alla ventina di saggi in Aristotle Transformed) offrono inoltre un'ampia materia per la discussione dei vari momenti specifici di questa storia. Le due raccolte curate da Sorabji hanno il merito non piccolo di rendere facilmente accessibili studi importanti - oltre ad alcuni inediti - che permettono di approfondire le avventure della filosofia all'ombra delle grandi scuole filosofiche dell'antichità.

Paulo Butti de Lima

Université de Bari paulo.buttidelima@uniba.it

\section{Laura SwIFT,}

Greek Tragedy. Themes and Contexts, London-Oxford-New York-New DelhiSydney, Bloomsbury, 20I6, I25 p., 14, 99 livres / ISBN 978-I-47423-684-6.

Cette étude que Laura Swift propose modestement aux collégiens ou aux étudiants débutants est en réalité une analyse très complète des ressorts de la tragédie grecque, de l'influence qu'elle exerça en son temps, et des leçons qu'elle peut encore nous offrir. Elle est distribuée en huit chapitres, consacrés successivement au genre tragique, aux trois grands auteurs, au mythe, aux héros, aux dieux, à la pensée contemporaine, aux avatars de la famille, et finalement au rôle si important du chœur. Une chronologie, un glossaire du grec et des termes techniques, des suggestions pour plus amples lectures, complètent cet intéressant volume.
La tragédie a pris naissance en Grèce vers la fin du VI ${ }^{\mathrm{e}}$ siècle av. J.-C. et a surtout fleuri au $\mathrm{V}^{\mathrm{e}}$ siècle, au moment même où se développait la démocratie. Aristote dans sa Poétique (I449a) relie l'éclosion de la tragédie aux improvisations propres au dithyrambe, sorte de chant choral en l'honneur de Dionysos. C'est d'ailleurs durant les Grandes Dionysies qu'étaient généralement présentées, en compétition, les pièces de trois poètes tragiques. La tragédie faisait donc partie d'une imposante célébration publique destinée à unir le peuple d'Athènes en l'invitant à réfléchir sur ce que signifiait le fait d'être Athénien, de vivre dans une famille ou dans une cité.

Laura Swift présente les trois grands auteurs de tragédies, Eschyle, Sophocle et Euripide, qui connurent un tel succès qu'Aristophane les imagine en compétition aux Enfers, dans une comédie jouée une année seulement après la mort de Sophocle. Presque toutes les tragédies évoluaient dans le monde du mythe, qui inspirait aussi les artistes en tous genres. Le public, vrai familier des récits mythologiques, était donc tout prêt à admirer, dans ce qu'il voyait sur scène, les qualités de la mise en œuvre. Au reste, le mythe était loin d'être immuable, comme en témoigne la variété des interprétations concernant l'histoire d'Hélène. La religion occupant une grande place dans la vie des Grecs, et la faveur divine envers une cité étant considérée comme essentielle à sa prospérité, on ne saurait s'étonner que les fêtes en l'honneur des dieux aient revêtu une telle ampleur. Comme le soulignait Robert Parker (Polytheism and Society at Athens, Oxford, 20o5, p. 136): “le théâtre était l'arène la plus importante dans la vie athénienne où la réflexion sur les questions théologiques s'exprimait en public ». Au V ${ }^{\mathrm{e}}$ siècle, Athènes était toute bruissante de spéculations intellectuelles; les sophistes y régnaient en maîtres. Une des principales questions débattues concernait le rôle de la culture et des habitudes sociales sur le comportement humain, quelle était la part 
de l'inné et de l'acquis. Autre matière à discussion : quelle était la différence entre le grec et l'étranger, qui était qualifié de « barbare " parce qu'il ne parlait pas le grec. Les Guerres Médiques avaient mis en contact ces deux mondes si proches dans l'espace mais si éloignés dans l'esprit. La tragédie d'Eschyle, Les Perses, produite en 472, huit ans après la victoire des Grecs à Salamine, évoquait la douleur des vaincus, regagnant leur patrie couverts de honte. Ainsi la tragédie rapprochait le public ordinaire des spéculations politiques ou philosophiques qui occupaient tant les intellectuels.

La tragédie dépeignait un monde où les sources normales de stabilité et d'autorité étaient mises en question. D'où la fréquence des problèmes familiaux et notamment du rôle dévolu à la femme dans la société athénienne. Même si elles ne prenaient aucune part à la vie politique, en tant que mères, elles engendraient des citoyens et des soldats qui assureraient la sécurité de la cité. En 45o, Périclès décréta que pour être citoyen, il fallait être né d'un père et d'une mère qui seraient tous les deux citoyens, ce qui rendait les Athéniennes indispensables à la survivance de la cité. La tragédie montrait clairement les méfaits de ceux, hommes ou femmes, qui dans le mariage ne se conduisaient pas comme ils le devraient. Comment reconnaître les qualités d'un homme, aussi bien dans la sphère familiale privée que dans la vie publique? Les relations pèrefils étaient bien souvent évoquées dans leur complexité : dans Edipe à Colonne, le père, maltraité par ses fils, se venge en les maudissant. La tragédie grecque se plaisait à dépeindre les dysfonctionnements de la famille: désintégration de ses formes traditionnelles, irrespect de ses membres pour leur propre rôle. Elle montrait les manques et les tensions inhérents au système social représenté, et en particulier les conséquences désastreuses de tout manque de respect envers les valeurs familiales.
En Grèce, toute pièce de théâtre, tragédie, comédie ou drame satyrique, exigeait la présence d'un chœur, groupe d'hommes ou de femmes qui chantaient et dansaient, tout en faisant des commentaires sur l'action qui se déroulait sous leurs yeux. Loin d'être un accessoire décoratif, le chœur est essentiel pour comprendre ce que signifiait la tragédie pour un auditoire grec. C'était lui qui devait guider le public, et mettre en lumière la pertinence des questions soulevées par le drame. Entrant généralement sur scène un peu après le début du spectacle, il y restait pendant toute sa durée, chantant des odes qui aidaient l'auditoire à comprendre les évolutions de l'action et donnaient aussi aux acteurs (ils étaient trois au maximum) le temps de changer leurs masques pour interpréter tel autre personnage. Une des principales missions de l'ode chorale était de relier l'intrigue de la pièce à de plus amples conceptions, et d'indiquer ainsi ce que l'auditeur pouvait apprendre en l'écoutant. Aussi serait-il particulièrement inopportun de minimiser l'importance des chœurs dans la tragédie grecque.

En résumé, c'est une évocation particulièrement pertinente de la tragédie grecque, de ses tenants et de ses aboutissements, que nous offre Laura Swift dans cet ouvrage fort attractif et joliment illustré.

Germaine Aujac

Université de Toulouse (UT2J) germaine.aujac@orange.fr 
Zara Martirosova ToRLone, Vergil in Russia. National Identity and Classical Reception,

Oxford University Press, “ Classical

Presences », 20I4, 3oo p. + 9 ill., 75 livres / IsBN 978-o-I9-968948-4.

En une suite de six chapitres, ce livre examine divers aspects de la réception du prince des poètes latins dans l'immense pays qui se dit héritier de Byzance. La thèse exposée est que cette réception est double : d'une part, il s'agit d'une réception “à l'Occidentale », qui adopte la lecture européenne de Virgile et l'utilise pour affirmer l'identité européenne de la Russie; d'autre part, d'une lecture proprement russe qui projette sur le poète des traits distinctifs autochtones.

Une longue introduction s'ouvre sur deux citations, de Pouchkine et de Blok, qui illustrent ces deux directions. C'est Pierre le Grand qui inaugure l'intérêt de la Russie pour Virgile, notamment par la création de l'Académie Slavo-gréco-latine, établissement scolaire où était dispensé aux élites un solide enseignement de langues classiques, en même temps que la rhétorique occidentale s'y imposait: en témoigne le De Arte poetica de Théophane Prokopovitch (I705); cela ne signifie pas pour autant un rejet de la littérature nationale, au contraire: ainsi L'Énéide apporte-t-elle un grille de lecture de l'histoire russe. Il n'existe pourtant pas de traduction canonique de L'Énéide qui se puisse comparer par exemple, à l'Iliade de Nicolas Gnieditch, ce qui fait dire à Mikhaïl Gasparov, la plus grande autorité en matière de littérature classique en Russie à la fin du $\mathrm{XX}^{\mathrm{e}}$ siècle, que “Virgile n'a pas eu de chance en Russie ». C'est encore l'opinion de Serguïeï Averintsev qui signale l'exception de Viatcheslav Ivanov, le "Virgilien russe ». Néanmoins les points de contact sont importants, et la « troisième Rome », la Rome orthodoxe, ne peut qu'être fascinée par le chantre de la première: c'est en lien avec elle que se forme la conscience russe, et dès le XVI ${ }^{\mathrm{e}}$ siècle, un portrait du poète romain a été représenté sur le mur occidental de la Cathédrale de l'Annonciation du Kremlin, ainsi que sur celle de la Dormition, et d'autres églises russes; Virgile est ainsi le témoin de la mission « romaine » de la Russie.

Le chapitre premier, intitulé «Virgile à la Cour ", s'intéresse à la promotion de Virgile à l'époque de Pierre le Grand, et à la première traduction de L'Énéide par Vassili Petrov. Virgile sert alors la gloire du souverain régnant et promeut la fierté nationale.

Le second, "Subversion et ironie », fait état, au contraire, de textes parodiques qui mettent à mal cette utilisation de Virgile aux fins de glorifier la dynastie des Romanov. Ce sont, par exemple, la pièce Didon de Iakov Kniajnine, et le poème burlesque L'Énéide à l'envers de Nicolaï Ossipov, inspiré de Scarron et d'Aloys Blumauer.

Le troisième chapitre est consacré à Pouchkine, dont le rôle absolument central dans la culture russe est d'emblée souligné. Non que Pouchkine fût un admirateur absolu de Virgile: mais il est le fondateur incontestable de la langue poétique russe. D'une immense culture, comme l'atteste sa bibliothèque qui a été conservée, il possédait l'édition Firmin Didot des Euvres de Virgile (I8I4), qui était son édition scolaire. Si l'on ne peut dire que l'influence de Virgile fut essentielle sur sa propre production, l'auteur propose néanmoins une lecture virgilienne du célèbre récit en vers “ Le Cavalier de bronze » (I833), aussi bien par le thème que par son traitement, avec comme point de départ les vers 29-34 du chant VII de L'Énéide : l'arrivée d'Énée dans le Latium et le projet de fonder une Ville, capitale de l'épopée nationale; à quoi Tchaïadev répondra dans ses Lettres philosophiques en i836 par le constat de l'orphelinage de la Russie dans la famille des nations.

Le chapitre IV traite de Virgile prophète messianique, donc, à partir de la réception 
en Occident de la quatrième Églogue, du rôle messianique de la Russie: de nombreuses spéculations à ce sujet se trouvent chez les poètes et philosophes de l' «Âge d'Argent » comme Vladimir Soloviev (Trois Rencontres, I899). C'est la théorie dite de “la troisième Rome » illuminée par la Sagesse, la Sophia inspirée à la fois par l'iconographie et la liturgie byzantines, le néoplatonisme, la gnose et la Kabbale, qui saurait réunir l'Orient et l'Occident sous la bannière du Christ, et assimilée à la Virgo du v. 6 de la quatrième Églogue. On aurait l'impression de perdre un peu Virgile de vue, si Soloviev n'avait entrepris en I887 une traduction de L'Énéide, en laquelle il voyait “ l'incarnation parfaite du principe romain d'universalité », ainsi que la confirmation de l'idée de la prééminence de Rome dans la conduite spirituelle du monde. Soloviev traduisit d'ailleurs également la quatrième Églogue, dont il partageait avec Ivanov l'interprétation messianique. Averintsev va aussi dans ce sens, en lisant très finement dans l'Églogue une « anticipation esthétique » du monde à venir (“ Deux mille ans avec Virgile»); une étude est réservée à la place de Virgile dans la Russie de l'émigration, et notamment, après qu'il eut quitté l'URSS, chez Viatcheslav Ivanov, disciple le plus proche de Soloviev et passionné par les rapports de l'Antiquité (étudiée à Berlin aux cours de Mommsen) et du christianisme. Rome où Ivanov s'installe et se convertit au catholicisme devient le symbole du but longtemps recherché.

Le chapitre V, consacré à Joseph Brodsky, fait apparaître un Virgile "postmoderne », chez qui gloire impériale et mission messianique sont remplacées par des thèmes mondains, voire triviaux. Enfin la conclusion fait le point sur les traductions russes du poète latin et s'arrête particulièrement sur celles d'Afanasi Fet et de Valery Brioussov. Suivent deux précieux appendices, qui sont des traductions en anglais, commentées, de l' “ Historiographie de Virgile » d'Ivanov et de «Sur Virgile » de Fedotov.
Au total, nous avons ici un livre qui éclaire tout autant le destin de Virgile que l'idée, assez mal connue des Occidentaux, que la Russie se fait d'elle-même: précieux pour notre temps.

Dominique MILLET-GÉRARD
Université Paris-Sorbonne
dominique.mg@wanadoo.fr

Jürgen von Ungern-STERNBERG,

Les chers ennemis. Deutsche und

französische Altertumswissenschaftler in

Rivalität und Zusammenarbeit,

Stuttgart, Franz Steiner Verlag, 20I7, 309 p.,

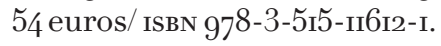

Sous un titre double, franco-allemand, et dans le cadre d'une entreprise triple, germano-helvéto-française, de quatre universités rhénanes (Bâle, Fribourgen-Brisgau, Mulhouse et Strasbourg), l'historien J. von Ungern-Sternberg, professeur émérite à Bâle, réunit quinze de ses travaux depuis I994, dont trois inédits. L'ensemble se clôt sur une liste des travaux de l'auteur, avec en particulier la thèse (I968-I970), sur le droit en situation d'urgence (Notstandsrecht) à l'époque républicaine tardive à Rome et l'habilitation (1975) sur Capoue à l'époque de la Deuxième Guerre punique.

La partie du titre en français peut surprendre, mais est expliquée dès l'avantpropos. En I870, Theodor Mommsen avait choqué l'opinion française par des “letttres ouvertes » à des Italiens. Après la paix de mars I87i, humiliante pour la France, il voulut remettre en marche au plus vite ses relations scientifiques avec Ernest Renan. Celui-ci répondit avec réserve, mais termina sur une note positive en citant Goethe et son refus du “patriotisme exclusif» et en priant son 
“ cher ennemi » Mommsen de continuer à voir en lui, malgré tout, un « ami ».

La définition donnée par Renan de la nation comme "principe spirituel », désir de vivre ensemble avec des souvenirs communs et " plébiscite de tous les jours », est de i882. Elle était censée contrer la définition de la nation par le philosophe Fichte, dès ı8o8, au plus fort de l'humiliation prusso-allemande face à la France napoléonienne, dans ses Discours à la nation allemande : la nation comme unité fondée sur une langue supposée originelle, pure et vivante, l'allemand étant selon Fichte très supérieur aux langues romanes dérivées du latin, et donc abâtardies et quasiment mortes à la naissance. Fichte n'envisageait cependant pas une unité à fondement ethnique, tous les peuples européens étant le résultat de «mélanges ». Mais nombre de ses successeurs plus ou moins légitimes, du XIX ${ }^{\mathrm{e}}$ au XX $\mathrm{XX}^{\mathrm{e}}$ siècle, du national-romantisme au national-socialisme, sommet paroxystique du processus, en passant par le pangermanisme et la « révolution conservatrice » des années ig2o et I93o, ne se sont pas privés d'inférer d'une langue « pure » et " supérieure » à une race tout aussi “pure » et « supérieure ».

C'est l'ambivalence des « chers ennemis » que l'auteur, à travers ses propres travaux, explore à propos des chercheurs allemands et français en sciences de l'Antiquité du XIX ${ }^{\mathrm{e}}$ siècle au XX' $\mathrm{XX}^{\mathrm{e}}$. Ces “ ennemis » deviennent ou redeviennent “chers " une fois la paix revenue, essentiellement par le biais de la coopération scientifique. On évite en général de traduire l'allemand Zusammenarbeit par “collaboration », terme négativement connoté depuis i940. Mais des personnages officiels et des journalistes français paraissent ne plus y prêter attention: simple inattention significative, voire révélatrice d'une forme d'inconscience historique?

Les deux premiers textes concernent ı9I4-I9ı8, l'antagonisme le plus virulent -
Bergson ne déclare-t-il pas en igí devant l'Académie des Sciences morales et politiques que la guerre contre l'Allemagne est « la lutte même de la civilisation contre la barbarie »? - cédant parfois le pas, même alors, au maintien d'une coopération trouvant cependant rapidement sa limite : dès I9I4, des chercheurs français de renom démissionnent de l'Académie de Berlin et de l'Institut allemand d'archéologie.

Les textes suivants sont plus focalisés : regards allemands sur Vercingétorix et français sur Arminius, le vainqueur de Varus dans la forêt de Teutoburg en 9 après J.-C. ; diversité franco-allemande des analyses de la “ clientèle » à Rome; approches différentes de l'histoire sociale de Rome, dont celle de Gaston Boissier, né en I823 à Nîmes, ce pôle de la romanité ; histoire pédagogique (Rome dans les programmes et ouvrages scolaires français et allemands de I85o à I9ı) et histoire des sciences (l'histoire ancienne à l'École française de Rome de 1873 à I940); Theodor Mommsen, son rapport à la France et à Strasbourg et la réception de ses écrits en France ; coopération franco-allemande sur les inscriptions de Délos; fin d'une amitié scientifique (Holleaux-Karo) à l'automne igı́. En guise de conclusion, la dernière contribution examine les conséquences de la guerre sur la communauté scientifique européenne.

Certains textes - mais pas tous, loin de làsont traduits dans « l'autre » langue. À noter l'heureuse présence d'un index des noms propres où se retrouvent plusieurs gloires comme l'inévitable Fustel de Coulanges, le limpide Camille Jullian, Marseillais de naissance et fondateur des “Antiquités nationales ", et le ferme républicain Claude Nicolet, lui aussi né à Marseille, et, sur la rive d'en face, drüben, Theodor Mommsen, Niebuhr et le toujours impressionnant Ulrich von Willamowitz-Moellendorf. Au total, un ouvrage certes un peu désordonné, mais où chacun, archéologue, historien, 
politologue, sociologue ou germaniste, puisera son miel.

Lucien Calvié

Université de Toulouse (UT2J) claude.calvie@orange.fr

Robert W. Wallace,

Reconstructing Damon:Music, Wisdom

Teaching, and Politics in Perikles'Athens, Oxford: Oxford University Press, 20I5, $223 \mathrm{p}$.,

64 livres / IsBN 9780I99685738.

This volume provides a systematic and accurately commented collection of Damon's fragments and testimonia, but, as a whole (as we will see), it is more than a simple collection. Damon of $\mathrm{Oa}$ was a musician, most likely versed not only in music in the strict sense of the word, but also in the wide range of interests and skills

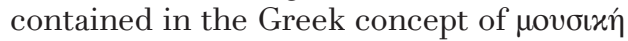
(an updated overview on this concept can be found now in P. Destrée-P. Murray [eds.], $A$ Companion to Ancient Aesthetics, Oxford: Wiley Blackwell, 20I5). He had a sound theoretical knowledge of music and, in some way, can be considered a 'philosopher of music.' As a matter of fact, he theorized the ethical influences of music, considering it capable of shaping the soul's virtues, thereby launching a philosophical tendency fated to endure for some centuries. Damon was the music teacher of Perikles; he played an active role in Perikles' cultural politics and for this reason, he was also ostracized from Athens. Therefore, in short, Damon was a leading figure in the cultural and, to a certain extent, political life of the Athens of the 5th century, even if his thought was destined to have a major impact on the field of music (for an updated analysis of the intellectual figure of Damon, I would like to refer to my contribution: Music and Philosophy in Damon of Oa, in: Ch. Vassallo [ed.], Presocratics and Papyrological Tradition, Proceedings of the International Workshop held at the University of Trier, 22-24 September 20I6 [= Studia Praesocratica], Berlin-Boston: De Gruyter, 20I8, forthcoming). His fame in antiquity was determined by a series of factors, most importantly by the authoritative judgement of Plato. As a matter of fact, in his dialogues (especially in the Republic) Plato often mentions Damon, showing the highest admiration for his musicological theories. In turn, the Stoic philosopher Diogenes of Babylon as well, who was deeply interested in music and particularly in its relationship with philosophy, agreed with (and developed) the core of Damon's theories on musical ethics. Since Diogenes of Babylon was the target of Philodemus' vicious attacks, especially (but not exclusively) in his treatise On Music, the Epicurean philosopher largely quoted Damon in his work. This is the reason why such an important part of the testimonia concerning Damon derives from the Herculanean papyrological tradition. Plato's favourable opinion of Damon is the main reason for his popularity in the Neoplatonic tradition, in particular in Proclus (on this point cf. E. Moutsopoulos, La philosophie de la musique dans le système de Proclus, Athens: Academy of Athens, 2004). But Damon was also long remembered in the musicological and scholiastic traditions, and we can find many references to him in the classical texts of historical and philosophical literature (the case of Plutarch is paradigmatic), in addition to the erudite tradition. His name still appears as an undisputed auctoritas in the field of music within the late antique 'encyclopedy' by Martianus Capella: the $D e$ nuptiis Philologiae et Mercurii.

The first group of fragments and witnesses on Damon was collected by Hermann Diels in his renowned Fragmente der Vorsokratiker, but various scholars have progressively enriched this first collection. In this respect, one cannot forget to 
mention the capital studies of U.von Wilamowitz-Moellendorff (Griechische Verskunst, Berlin: Weidmann, I92I) and F. Lasserre (Plutarque, De la musique: Texte, traduction, commentaire, précédés d'une étude sur l'éducation musicale dans la Grèce antique, Lausanne \& Olten: Urs Graf, ig54). Lasserre also took a series of Platonic texts into account, in which no direct reference to Damon appears, in his collection. Other scholars have published a selection of the most important texts, providing them with useful commentaries, as in the collection of the Pythagoric testimonia by M. Timpanaro Cardini (Pitagorici: Testimonianze $e$ frammenti, vol. III, Florence: La Nuova Italia, I958).

Robert W. Wallace has now carried out a new collection of the documentary material concerning Damon. In this praiseworthing collection, only one Herculanean testimonium seems to have been left out: Philod., Rhet. IV, P.Herc. IIо4 (olim III4), fr. 7 Vassallo (= II, p. 299 Sudhaus = deest in DK et in Lasserre). The new reconstruction of this testimonium, in which Perikles is mentioned as a pupil of both Anaxagoras and Damon, will appear in the Corpus of the Herculanean testimonia to Presocratic philosophers edited by $\mathrm{Ch}$. Vassallo $(\mathrm{CPH}$ [= Corpus Praesocraticorum Herculanense] $\mathrm{X}$ 40, in: Ch. Vassallo, The Presocratics in the Herculaneum Papyri: Texts, Translations, and Commentary [= Studia Praesocratica], Berlin-Boston, De Gruyter, in preparation). This passage does not appear even in the Index Praesocraticorum Philosophorum Herculanensis (IPPH) edited and recently published by Vassallo as well (A Catalogue of the Evidence for Presocratics in the Herculaneum Papyri, in: "Archiv für Papyrusforschung", 62/1 [2016], p. 78-108). It is an addendum which the young Italian scholar brought to my attention and that, for obvious chronological reasons, Wallace could not have known from current secondary literature.
The form adopted by Wallace for his volume is not that of a classical Sammlung der Fragmente, but more similar to Lasserre's choice, although the positions of the two scholars are quite different. In fact, the first part of the volume is a kind of monograph (p.3-Io4), followed by the second part, the collection of testimonia and fragments (p. I07-I8I); at the end we find four Appendices (p. I83-205) that mainly refer back to the first monographical part. This peculiar structure provides, on the one hand, the reader with a large treatment of the topic in which Wallace is mainly interested: the reconstruction of Damon's personality within an inquiry on music, cultural teaching, and politics in the Athens of Perikles. But on the other hand, it has its limits: the collection of fragments seems to be, to a certain extent, a confirmation of the theses expounded in the first part, whereas an autonomous and accurate analysis of the texts should be the starting point for any historical and cultural reconstruction.

Among the various theses, there is one in particular that Wallace cares about: the inexistence of the Damon's work known by some traditions with the title of Areopagiticus. Now, it is well known that Wilamowitz considered the Areopagiticus the first example of Attic prose. According to H. Ryffel (Eukosmia: Ein Beitrag zur Wiederherstellung des Areopagitikos des Damon, in: "Museum Helveticum", 4 [I947], p. 23-38), this work would have influenced, to some extent, the Areopagiticus of Isocrates as well. Wallace's thesis overturns a fairly consolidated trend in the scholarship. In defense of his view, the American scholar uses significant argumentative variations. Sometimes Wallace seems to intend to demonstrate that Damon's Areopagiticus never existed; in other cases, he tries to show that no work of Damon ever existed, or he wonders whether he ever wrote something: cf. Appendix I (p. I83-ı85), where Wallace concludes: "in publishing nothing Damon was by no means unique among even his 
younger contemporaries" (p. 185). All in all, I think that the attempt to demonstrate this thesis has failed. In its support, Wallace uses a hypothesis already held by $\mathrm{H}$. Bücheler (Oi

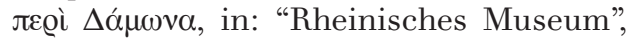
40 [1885], p. 3og-3І2), but at the same time he deeply misconstrues it. According to Bücheler, Philodemus' source in Mus. IV, P. Herc. I497, coll. I47, 34-I48, 5 Delattre, II, p. 3og-3 Io $(=37$ B 2 DK = fr. 5 Lasserre = B I3 Wallace $=I P P H$ IX 3o) was neither a work by

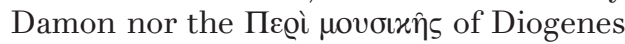
of Babylon (who, however, surely quoted Damon in his treatise, remembering his doctrines: cf. Diog. Bab., SVF III, fr. 56), but a supposed dialogue of Heraclides Ponticus, whose main character would have been Damon. But Wallace's conclusion does not agree with that of Bücheler, who admitted the existence of a Damon's work (along with the alleged dialogue by Heraclides Ponticus). At the same time, Wallace is also in disagreement with Philippson, who, on the one hand, hypothesized that the only source for Damon in Philodemus' On Music was Heraclides, but, on the other, never questioned the existence of Damon's Areopagiticus. In contrast, Wallace maintains that all we know on Damon via Philodemus would derive from a supposed writing by Heraclides (whose existence remains totally hypothetical) and that Damon's Areopagiticus never existed.

So that begs the question: if - as Wallace claims - this writing never existed, what source did Heraclides use to write his dialogue (of course, assuming that this dialogue existed), in which Damon figures as a character and his theories were exposed? Wallace's hypothesis detroys itself along with the entire Damonian tradition: but a hypothesis that ends up destroying the same tradition it should explain seems to be methodologically unacceptable. Not to mention, Heraclides Ponticus never mentions Damon in his extant fragments. This being the case, the supposition that he wrote a dialogue devoted to Damon and referred in it his doctrines is totally groundless. Furthermore, thanks to Diogenes Laërtius (V 86-88), we have a catalogue of Heraclides' works. This list

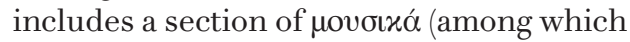
a treatise On Music in two books is also taken into account), but none of these titles refers to Damon.

In reality, the existence of a work of Damon belonging to a Preplatonic era (I consider here a secondary point whether this work was written in the form of an Areopagiticus or not), is shown by the most important of the sources on Damon at our disposal: viz. Plato. One should remember

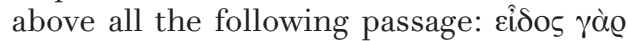

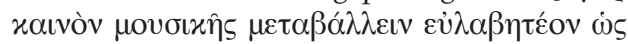

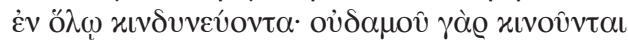

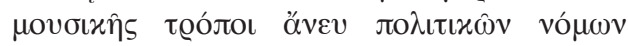

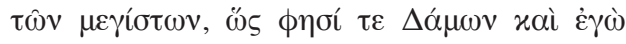

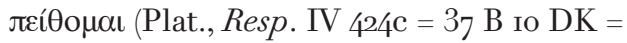
$\mathrm{B} 2$ Wallace). This is a clear and emphasized quotation of Damon's work. It should be added that in the famous section on rhythm Plato also mentions Damon with regard to his opinions on the topic with great approval (Plat., Resp. III $400 \mathrm{a}=37 \mathrm{~B} 9 \mathrm{DK}=$ B I Wallace). Many scholars maintain that in the section on the ópovía Plato also took Damon's work into account: see, in particular, chapter III (entitled Damone e $i$ sofisti) of A. Barker's book Psicomusicologia nella Grecia antica (ed. by A. Meriani), Naples: Guida, 2005, p. 57-74, who considers it highly likely that Damon was the source of the Platonic treatment of harmonies in the Republic (p. 67). In any case, I think I have already demonstrated that Plato does not blindly follow or uncritically accept Damon's theories, but he introduces instead own significant innovations: cf. A. Brancacci, Musique et philosophie en République $I I-I V$, in: M. Dixsaut (ed.), Études sur la République de Platon, vol. I : De la justice, Paris: Vrin, 2005, p. 89-Io6.

Another important element for reconstructing the intellectual figure of Damon is the explanation of his relationship with 
Perikles. To this biographical event the question of ostracism is closely connected. According to Wallace, Damon was really banished from Athens: i.e. the ostracism was a historical fact and should not be doubted as some scholars have done on the basis of arguments and testimonia that he correctly reports and discusses (p.53-55). Wallace pays due attention to all these problems, along with the thorny question of Damon's chronology. On this last point, two different theories are in competition: as a matter of fact, some scholars maintain that Damon was born "in the early fifth century, perhaps c. 5oo"; others instead postdate his birth "a generation after 5oo" (p. I86), extending his activity untill the last years of the $5^{\text {th }}$ century. Wallace devotes the large and well-documented Appendix 2 (p. I86-ig3) to this problematic question. He presents all the elements that could support the early chronology, towards which he leans. As for his ostracism, it is unfortunately impossible to determine a definite date, grounded on objective factors: therefore, Wallace rightly limits himself in concluding that "sometime between 445 and 44I - most probably in 442 - Damon was ostracized" (p. I93). On the cultural level, Wallace tries to associate as much as possible Damon with the Sophists (p. I9-2I). Now, in regard to this problem, in my view we must definitively settle that Damon was not a sophist in the proper sense, but only a member of 'Perikles' Circle': that is to say, a movement historically (and theoretically) earlier than the birth of the Sophistic movement and far removed from the development of the sharp distinction between 'sophist' and 'philosopher.' This distinction, as we know, established itself after the harsh antisophistic polemics of Plato, probably already held by Socrates and than variously adopted by other Socratics like Antisthenes. The 'Perikles' Circle' represents a capital event for the cultural history of Athens of $5^{\text {th }}$ century. In this circle, not only different philosophical stances (for instance, Anaxagoras, as the representative of physical research; Socrates, who probably assumed an independent and critical position; Protagoras, who would launch the Sophistic movement; Phidias and Damon,

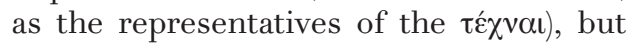
also various political orientations (Socrates, for example, surely did not share the prodemocracy tendencies of this circle) lived miraculously together.

In conclusion, Wallace's book provides a lot of material and food for thought. Although not all his theories seem to be acceptable, the accuracy of the volume is commendable. The secondary literature quoted and discussed is wide; his new collection of Damon's fragments and witnesses was a desideratum, and it is really welcomed in the scholarship on Damon; finally, in spite of the lack of clear and abundant testimonia, his attempt to demonstrate the great relevance of Damon's figure in his historical period seems to have been successfully realized.

Aldo Brancacci

Università degli Studi di Roma 'Tor Vergata aldo.brancacci@uniroma2.it

T. P. WisEman,

The Roman Audience: ClassicalLiterature as Social History,

Oxford, Oxford University Press, 20I5,

$327 \mathrm{p}$.,

75 livres / ISBN 9780198718352.

L'ouvrage de T.P. Wiseman s'inscrit dans les nombreuses études consacrées à la culture populaire et aux transferts culturels dans l'Antiquité, même s'il ne le dit pas explicitement. Dix chapitres ont ainsi pour but de cerner le rôle et la place du public dans toute la littérature romaine. L’approche est délibérément diachronique pour mesurer toutes les évolutions.

Le premier chapitre (Times, Books and Preconceptions) est un préambule (p. I-9) 
sur la nature de la littérature, en intégrant le support d'écriture, la longue durée et le statut des textes. Il faut tenir compte du contexte social et culturel de production littéraire. Selon l'auteur, la littérature n'était pas réservée à une élite lettrée, comme l'attestent par exemple les pièces de théâtre jouées en public. Cette position invalide les oppositions simplistes entre les élites et le peuple.

Les chapitres 2 (Rome before literature) et 3 (Dionysus and Drama) cherchent à saisir le climat culturel de la Rome archaïque, de la création du Forum au VII ${ }^{\mathrm{e}}$ s. av. J.-C. à l'utilisation du papyrus au $\mathrm{III}^{\mathrm{e}} \mathrm{s}$. av. J.-C., soit en grande partie avant l'apparition de la littérature latine. L'approche est ici résolument comparatiste, car l'auteur s'attarde sur la littérature grecque, notamment sur l'œuvre d'Homère. D'ailleurs les interactions et les transferts culturels du monde grec vers Rome sont anciens : Tarquin le Superbe prétendait descendre d'une lignée d'aristocrates corinthiens.

Le manque de sources littéraires implique aussi le recours à toutes les sources, comme la statuaire, les vases grecs ou les miroirs gravés. Après l'expulsion des Tarquins, l'introduction à Rome du culte de Cérès, Liber et Libera aurait été influencée par celui de Dionysos Eleuthereus à Athènes. Il aurait par conséquent pu s'accompagner de représentations théâtrales. Une récente découverte archéologique dans la cité étrusque de Caere a mis au jour un protothéâtre datant du $\mathrm{V}^{\mathrm{e}} \mathrm{s}$. av. J.-C., prouvant l'existence de ces structures. Le troisième chapitre se focalise sur les Ludi. En 338 av. J.-C., G. Maenius fit aménager une galerie sur le Forum, ce qui pose la question de la nature des spectacles donnés et du public. Concernant les Ludi Romani, l'apport des fragments de Fabius Pictor et Gnaeus Naevius sont déterminants. Les Jeux de 240 av. J.-C. mettent alors en lumière les transferts culturels en vigueur, en intégrant la tradition dionysiaque grecque et l'utilisation du papyrus égyptien.
Le chapitre 4 (An Enclosure with Benches) s'intéresse plutôt aux ludi scaenici grâce à Plaute et Térence. Toutefois un autre problème émerge car le contexte matériel des représentations reste trop inconnu. C'est pourquoi l'auteur s'intéresse à différentes structures (theatrum, scaena, cauea) pour faciliter la diffusion au public. Ensuite le chapitre 5 (Makers, Singers, Speakers, Writers) traite notamment des uates et des récitations publiques de tradition grecque. T. P. Wiseman en profite pour remettre en cause les recherches récentes focalisées sur les élites. Dans cette perspective, l'apparition de la littérature latine aurait été l'agent de l'acculturation aristocratique et l'épopée “was written for and eventually written by the Roman elite whose education best equipped them to reap literature's reward ». La place du peuple est ici a contrario prise en compte, notamment lors des funérailles aristocratiques et de l'éloge funèbre. Cependant selon l'auteur, l'équilibre entre les riches et les pauvres a été remis en cause par l'arrogance aristocratique et les violences de la fin de la République. L'aristocratie corrompue fut donc fustigée par Lucilius et Varron dans leurs satires. Dans le chapitre 6 (A Turbulent People), l'auteur soutient également l'idée que le peuple romain a soutenu des politiciens comme Pompée contre l'arrogance aristocratique. Les troubles politiques de la fin de la République mettent alors en exergue l'implication du public romain. Contre l'idéologie aristocratique, Pompée fit construire le premier théâtre permanent à Rome.

Les chapitres 7, 8 et 9 (Rethinking the Classic) sont conçus comme un triptyque s'intéressant à la période 59 - 42 puis $42-28$ et 28-8 ap. J.-C., une période de foisonnement littéraire, marquée par des guerres civiles et l'instauration du Principat, très bien connue dans l'historiographie. La question de la diffusion de l'œuvre est posée, notamment pour un poète comme Catulle qui eut droit à 
des funérailles publiques. Le peuple devait par conséquent le connaître. Le Principat change progressivement la donne et le peuple dispose dorénavant de plusieurs structures permanentes de spectacle. L'époque augustéenne est marquée par l'importance des cercles littéraires et l'apparition de l'élégie. La communication politique est aussi accaparée par le princeps, tandis que les sénateurs, comme Asinius Pollion, sont réduits à inviter des citoyens chez eux pour des lectures publiques.

Enfin le dernier chapitre (Under the Emperors) s'intéresse logiquement à l'évolution du public dans la longue durée impériale, " the political story of an arrogant oligarchy tamed by the Caesars ». L'auteur passe ici en revue les différents genres, en précisant que les classiques étaient alors récités au théâtre. L'auteur termine par la critique chrétienne des ludi scaenici en quelques pages (p. I80-I82). La vision chrétienne aurait gagné à être plus développée pour tenir compte de l'ensemble de l'histoire romaine.
Afin de s'adresser à un large public, T. P. Wiseman défend le parti-pris d'avoir rejeté toutes les notes de bas de page à la fin de l'ouvrage. Certes le but est louable, mais cela rend la lecture plus difficile avec des va-et-vient incessants. C'est d'autant plus dommage que les notes sont très conséquentes et couvrent une partie substantielle de l'ouvrage (p. i83-29i).

Pour conclure, ce livre apporte une contribution aux études consacrées à la culture populaire et à la place du public dans la littérature antique. Les passages sur la Rome archaïque sont les plus éclairants. En dépit de quelques approximations mineures et d'oublis, l'opposition récurrente entre le peuple et l'arrogance de l'aristocratie aurait cependant gagnée à être nuancée.

Cyrielle Landrea

Université Lille-SHS

cyrielle.landrea@orange.fr 
